\title{
PETROLOGY AND GEOCHEMISTRY OF INTRUSIVE ROCKS FROM THE SOUTH OF NAQADEH, WEST AZERBAIJAN, IRAN
}

\author{
Abdollah Kordi ${ }^{1}$ \\ Afshin Ashja-Ardalan ${ }^{2}$ \\ Seyed-Jamal Sheikhzakariayi ${ }^{3}$ \\ Nasser Ashrafi ${ }^{4}$
}

\begin{abstract}
The intrusive rocks of Naqadeh are features of Laramide magmatism in the Sanandaj-Sirjan zone. According to petrographic studies, the composition of intrusions consist of acidic, intermediate, and basic rocks. including syenogranites, monzogranite, granodiorites, quartz diorites, quartz monzonites, quartz monzonite, quartz syenite, and olivine gabbro-in order of abundance. Minerals forming intrusive rocks include alkali feldspar (orthoclase and microcline), quartz, plagioclase (andesine to anorthite), amphibole (magnesiohornblende to tschermakite hornblende), pyroxene (diopside), olivine (hyalosiderite to hortonlite), apatite, titanite, zircon, muscovite, and
\end{abstract}

opaque minerals. The chemical analysis of the granitoids revealed their potassium-rich calc-alkaline nature, falling within the meta-alumin to peralumin range in terms of alumin saturation. Tectonomagmatic diagrams for the studied rocks suggest that they are compatible with granitoids of the continental-subduction zone. Primitivemantle- and chondrite-normalized spider diagrams indicated no depletion in any elements, exhibiting a negative overall slope which is consistent with subduction patterns (negative $\mathrm{Ta}, \mathrm{Ti}, \mathrm{Zr}$ anomalies, and positive $K$ anomaly). Overall, a review of field observations, mineralogy, geochemistry, and diagrams illustrating the sources of granites

\footnotetext{
${ }^{1}$ Ph.D. Student in Petrology, Department of Geology, North Tehran Branch, Islamic Azad University, Tehran, Iran.

${ }^{2}$ Assistant Professor, Department of Geology, North Tehran Branch, Islamic Azad University, Tehran, Iran.

${ }^{3}$ Assistant Professor, Department of Geology, Science and Research Branch, Islamic Azad University, Tehran, Iran.

4 Assistant Professor, Department of Geology, Payame Noor University, Tehran, Iran. Corresponding author: afshinashjaardalan@yahoo.com.
} 
showed that the intrusive rocks in the study region are, in fact, I-type granite and that the magmatism is a result of the subduction of the oceanic crust, Neotethys, under the Iranian continental crust.

Keywords: Naqadeh Intrusive Rocks, Sanandaj-Sirjan Zone, Petrography, Minerals Chemistry, I-Type Granite.

\section{Introduction}

The study region stretches northeast of Iran in West Azerbaijan Province and is part of the northwestern deformation and ophiolite belt of the Sanandaj-Sirjan zone (Fig. 1) based on the classification of structural units (Stocklin, 1968) and structuralsedimentary zones (Aghanabati, 2004) of Iran. The zone $(150-250 \mathrm{~km}$ wide and $1500 \mathrm{~km}$ long) features one of the most complex structures in Iran (Alavi, 1994) and houses the Zagros Mountains at its center (Sengor, 1990). Even though the age, nature, and source of these rock masses remain unclear, they provide essential information as regards the geological history of the SanandajSirjan zone. It is widely believed that the rock masses are mainly of the calc- alkaline granitoid type that formed during the subduction of the Neotethys under the Iranian plate (Sepahi Garw, 2007; Ahmadi Khalaji et al., 1999; Ghalamghash et al., 2009; Yazdi et al., 2017; Khodami and Kamali Shervedani 2018; Novruzov et al., 2019) and following the collision of the Iranian and Arabian Plates. A specific time scale is yet to be presented for these events, but there is a consensus that the subduction process began during the Middle Jurassic. However, the exact time of the continental collision remains in dispute with some authors dating it back to the Late Cretaceous (Berberian and King, 2000; Mohajjel and Fergusson, 1981), whereas others attribute it to the Eocene (Numan, 2000; Ghasemi and Talbot, 2009; Mazhari et al., 2006) and even Miocene (Mohajjel et al., 2003; Omrani et al., 2008). Nonetheless, in the study region, granitoid intrusive rocks from the Late Cretaceous are abundant that penetrated older formations (limestones and Upper Cretaceous limestone dolomites) during Laramide orogeny. Contact metamorphism takes place at the intersection between the rock mass and country rocks resulting in the formation of dolomite- and calcite-rich Hornfels. As the final results of the granitic magma 
sap, several white veins, reaching several meters in thickness, penetrated various parts of country rocks from Precambrian to Cretaceous, as a sign of the last magma injection phase in the region.
Given their intrusive nature, the enclaves of the study region feature darker tones, curved and round shapes with a concentration of cognate enclaves (autolith) southeast of the area.

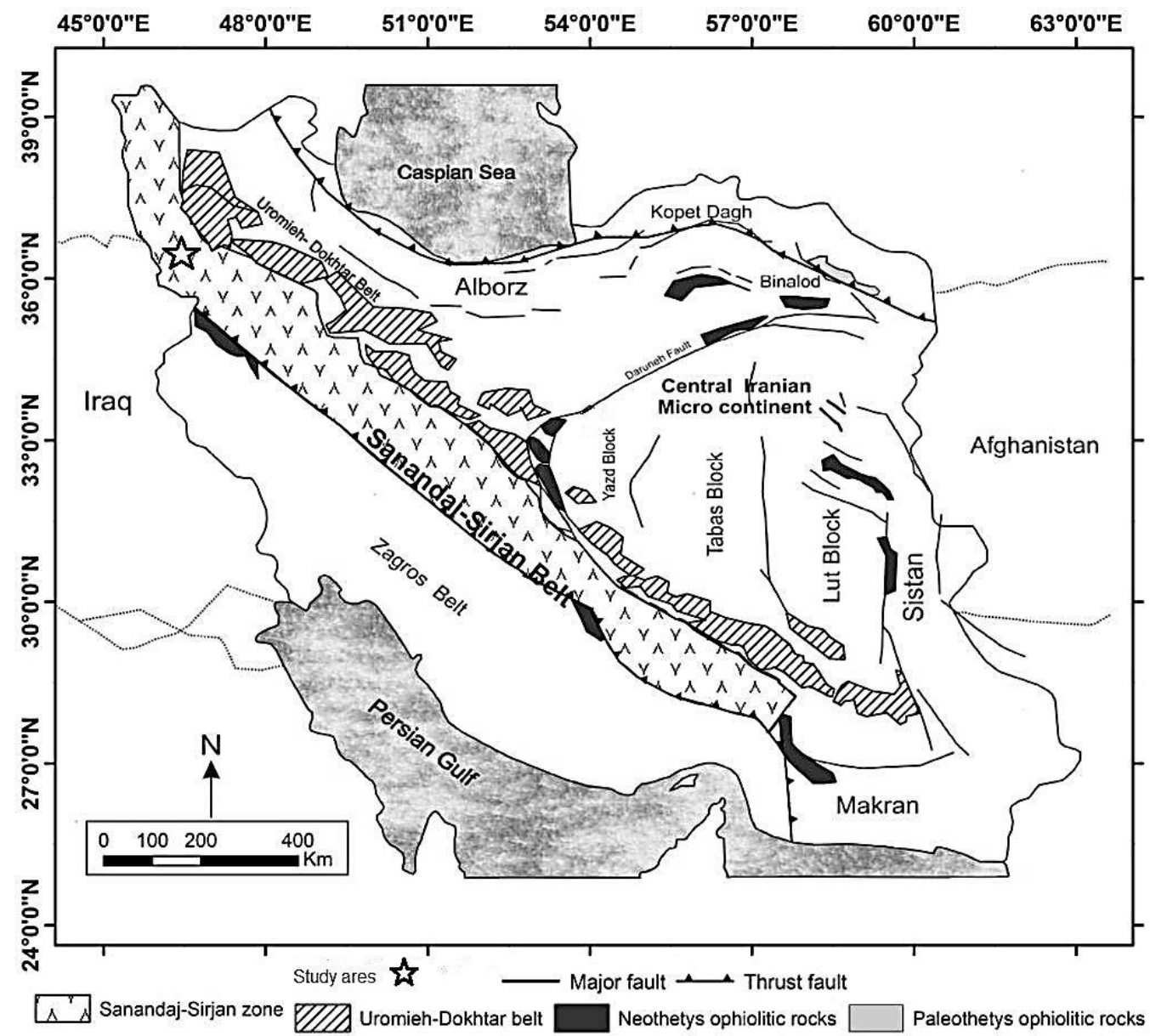

Figure 1. Geology of Iran (Courtesy of Aghanabati, 2004). Structural-sedimentary zones of Iran.

\section{Geology}

Stretching across $1200 \mathrm{~km}^{2}$, the study region spans between the $45^{\circ} 15^{\prime}$ and $45^{\circ} 30^{\prime} \mathrm{E}$ and $36^{\circ} 30^{\prime}$ and $37^{\circ} 00^{\prime} \mathrm{N}$ coordinates, $7 \mathrm{~km}$ south of Naqadeh, 30 $\mathrm{km}$ east of Piranshahr, and $39 \mathrm{~km}$ west of Mahabad (Fig. 2). The oldest rock outcrops in the region include a 
collection of metasomatic rocks with greenschist facies and traces of igneous rocks that are part of an anticline $10 \mathrm{~km}$ east of Naqadeh. The Cambrian deposits include Barut, Lalon, and Mila formations on top of the said collection. Permian carbonate deposits were pushed above the said younger deposits by thrust faults. Although the large stratigraphic gaps date back to the Ordovician, Silurian, Devonian, and Carboniferous, there is no trace of deposits from these periods in the region. On the other hand, dolomites and dolomite limestones of the Ruteh formation from The Permian are extensively common in the region, although the bases of rocks from this period do not crop out. As part of the Sanandaj-Sirjan zone, the study region has been considerably influenced by the phenomena and events occurring in this zone, particularly in the northern parts. Cretaceous rocks are common in the area spreading mainly south of the study region. The Cretaceous sequence comprises green to gray shales and gray limestone. Based on the positioning of the upper and lower formations, the sequence belongs to a period from the Lower Cretaceous to the Late Cretaceous. However, only evidence from the Late Cretaceous can be found in the study region. The broadest Cretaceous unit in the region is the one corresponding to the Late Cretaceous that holds shale and gray, slate, schist, and mica sandstones with pen erosion and thick and thin limestone strata in most places. 


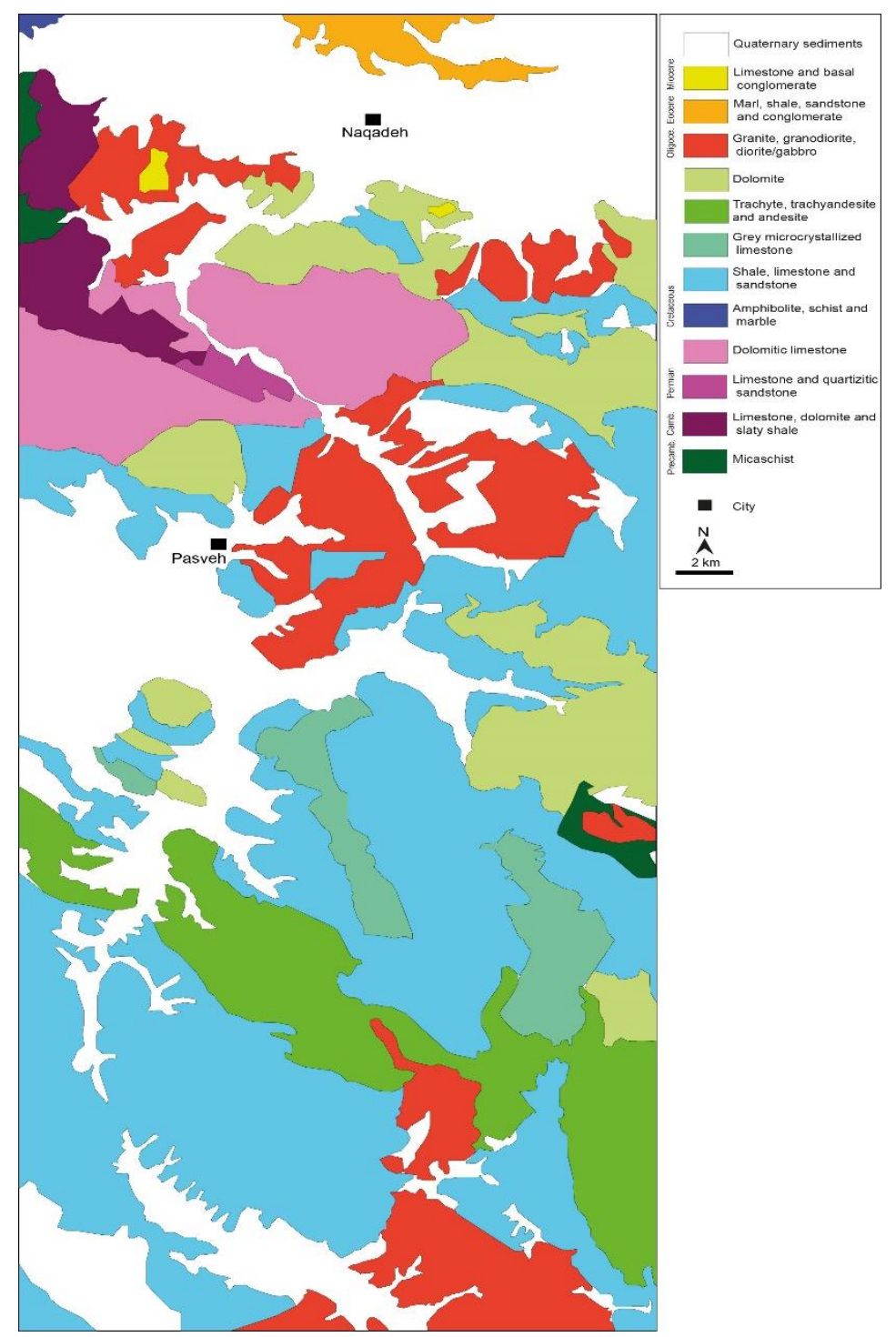

Figure 2. Geologic map of the study region (adapted from the 1:100,000 map of Naqadeh)

\section{Materials and Methods}

Field studies were undertaken first, and various outcrops and/or outcrops with unique relationships with their surroundings were sampled. During the 14-day field expedition, a total of 248 samples were collected from various parts of the region for lab investigation,
126 of which were used for preparing thin sections. After a preliminary microscopic investigation, 20 suitable samples were submitted to Kansaran Binaloud Co. for XRF and ICP-MS chemical analyses, the results of which are presented in the appendix (Tables 1 and 2). Of the 20 samples, 10 were 
selected for an additional microprobe analysis to obtain a more accurate chemical analysis of the minerals. Sixtyeight points of the samples with plagioclase, amphibole, pyroxene, and olivine minerals were analyzed by the CAMECA WDS SX100 Electron Probe Microanalyzer at $20 \mathrm{kV}$ accelerator voltage and $10 n A$ electric current intensity, with the results presented in the appendix (Tables 3-6). The analysis results were evaluated by GCDkit, Minpet, and Microsoft Excel.

\section{Petrography and Chemistry of Minerals}

\section{Petrography}

Based on petrological studies and the modal naming of rocks, the study region features acidic, intermediate, and basic intrusive rocks. These components come with various petrologies including syenogranites, monzogranite, granodiorites, quartz diorites, quartz monzonites, quartz monzonite, quartz syenite, and olivine gabbro-in order of abundance. The most noticeable feature of the felsic parts of the Naqadeh intrusive rocks is their many silica veins and the expansive fine-grained mafic enclaves that indicate the partial intermixture of mafic magma with felsic magma to create granitoid-forming magma (Fig. 3). The mineralogy of the intrusive rocks in the study region includes light, semi-crystalline to amorphous alkali feldspar with microcline and perthitic orthoclase in the form of coarse phenocrysts scattered in a fine-grained matrix, and traces of semicrystalline and round to amorphous quartz phenocrysts with clear crystals filling voids in some sections-but mainly non-crystallized microcrystalline in thin sections. In addition, crystalline to semi-crystalline plagioclases with albite, albite-carlsbad, and albite-pericline with regular and alternative local structures are present in these rocks. Dark minerals include amphibole, pyroxene, and olivine. Moreover, apatite, titanite (sphene), zirconia, muscovite, and opaque minerals were found in some sections in smaller amounts (Fig. 4). Most of the rock texture in the study region consists of hypidiomorphic granular to intergranular textures and, to some extent, granophyric, myrmekitic, perthitic, and poikilitic textures. 


\section{Gênerg.
Direitox}

Periódico do Núcleo de Estudos e Pesquisas sobre Gênero e Direito

Centro de Ciências Jurídicas - Universidade Federal da Paraíba V. 8 - No 03 - Ano 2019

ISSN | 2179-7137 | http://periodicos.ufpb.br/ojs2/index.php/ged/index

\section{0}
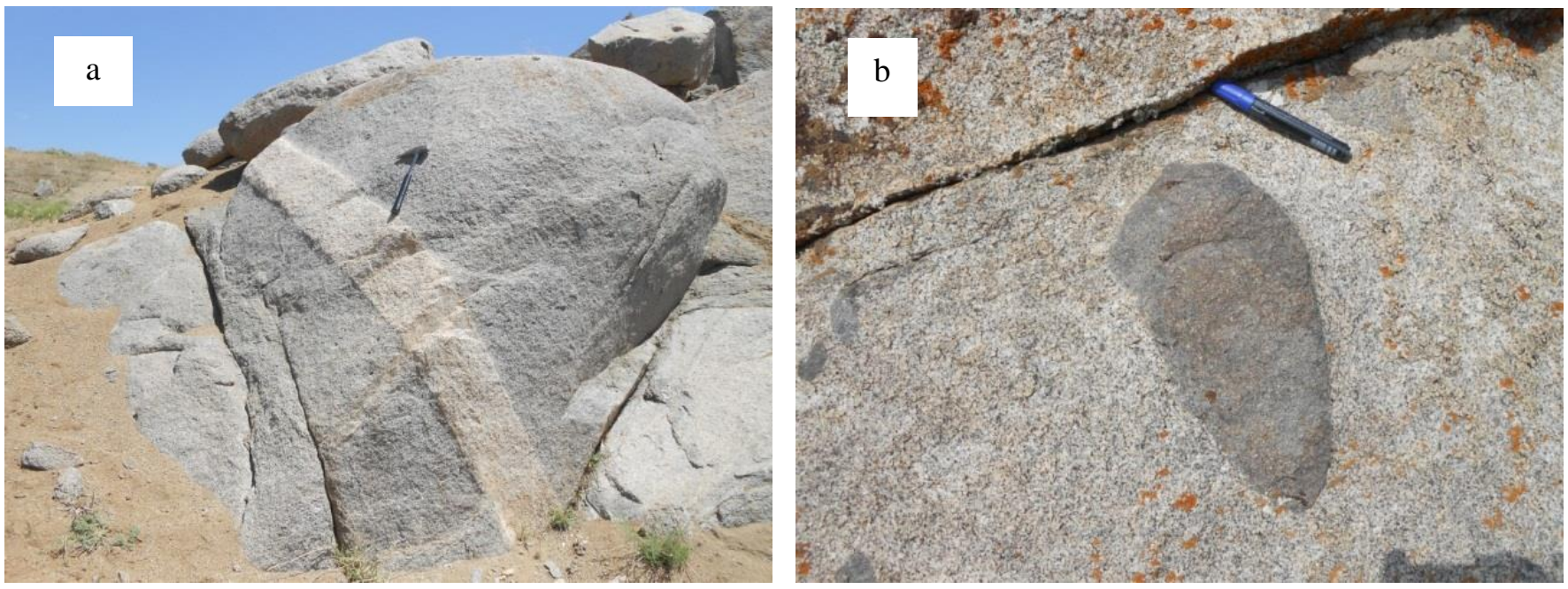

Figure 3. a) 20-cm-thick silica veins in granitoid rocks (northeast view); b) dark autolith with sharp boundaries with the local granitoid.
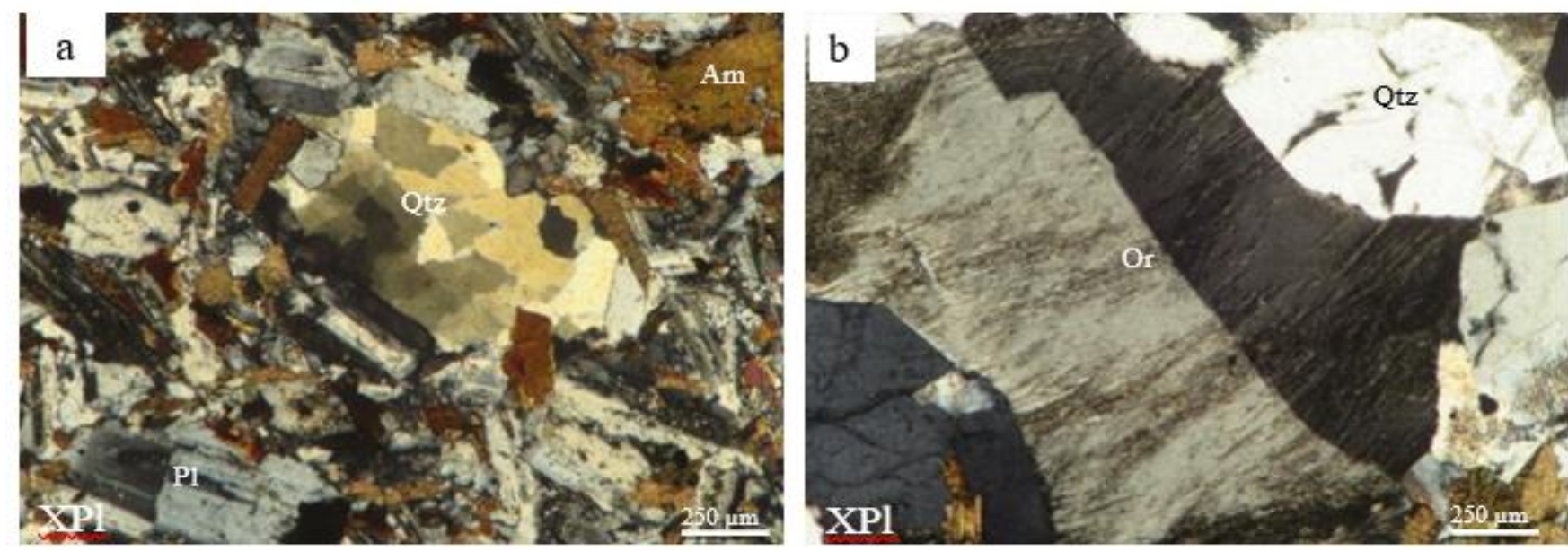


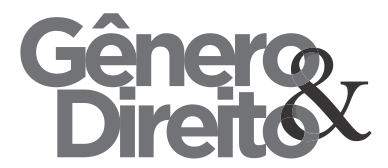

Periódico do Núcleo de Estudos e Pesquisas sobre Gênero e Direito

Centro de Ciências Jurídicas - Universidade Federal da Paraíba V. 8 - No 03 - Ano 2019

ISSN | 2179-7137 | http://periodicos.ufpb.br/ojs2/index.php/ged/index
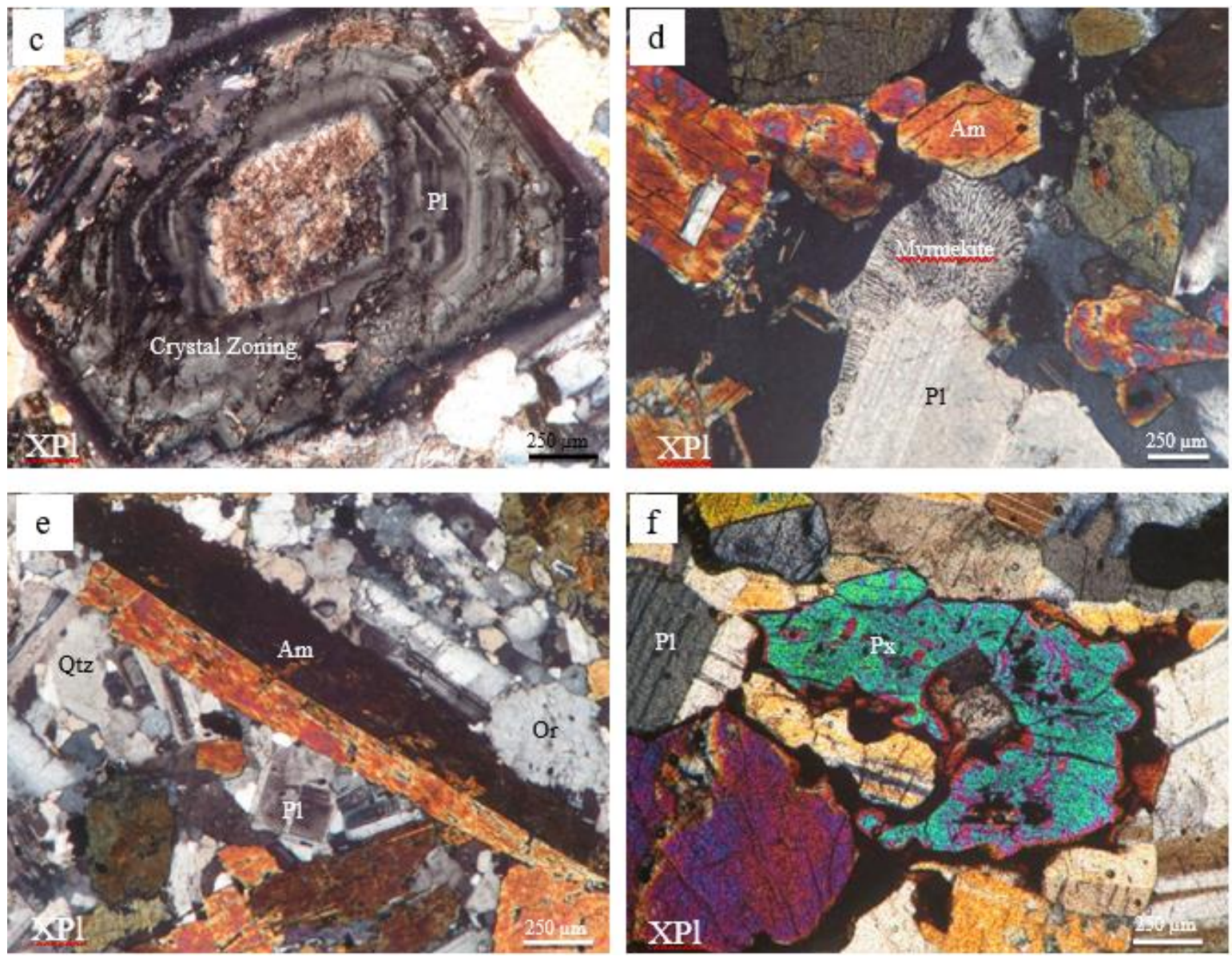

g

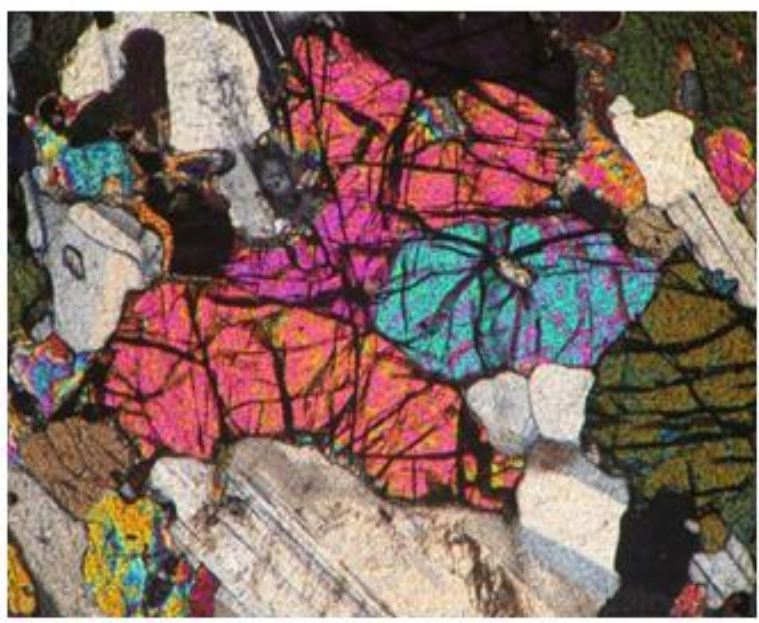

Figure 4. a) Cumulative quartz crystals with undulose extinction in syenogranite; b) coarsegrained orthoclase with carlsbad in monzogranite; c) plagioclase with alternative zoning decomposing from the center in granodiorite; d) myrmekitic microstructure at the edge of plagioclase beside automorphic amphibole in granodiorite; e) stretched amphibole phenocrysts 
with carlsbad in monzodiorite; f) uralitized augite phenocrysts with dissolution rims in gabbro; g) olivine grains as inclusions on the plagioclase in gabbro; All images were taken under $X P L$ lighting.

\section{Chemistry of Minerals}

The albite (Ab) $\mathrm{NaAlSi}_{z} \mathrm{O}_{8}$ anorthite (An) $\mathrm{CaAl}_{2} \mathrm{Si}_{2} \mathrm{O}_{8}, \quad$ and orthoclase (Or) $\mathrm{KAlSi}_{2} \mathrm{O}_{8}$ charts were used to name the feldspars (Deer et al., 1992). According to the results of chemical point analysis, in these rocks, the average plagioclase composition is $A n_{73.82} A b_{24.04}$ and $O r_{2.13}$, which falls in the andesine-anorthite range. The broad composition spectrum of plagioclases can be the result of changes in the melt composition and the vapor pressure of water and other fluids, leading many plagioclases to take up alternative zoning with different compositions at the rim and the center of the crystal.

The microprobe analysis results are suggestive of the calcic nature of the amphiboles in the studied intrusive rocks (Hawthorne, 1983) that can be classified as magnesiohornblende and tschermakite hornblende with an average silica content of less than 7.3. According to Leake et al., 1997, amphiboles containing over 7.3 silica are the result of subsolidus processes (Chivas, 1982; Agemar et al., 1999).

Pyroxenes are more resistant than amphiboles and are less affected by alteration, and their composing elements represent those in the magma. Based on the point analysis results, the average pyroxene composition in the study region is $W_{049.94} E_{28.85} F_{21.19}$ and falls in the diopside range (Morimoto et al. 1988).

The structural formula of olivine was represented by the general formula $(\mathrm{Mg}, \mathrm{Fe})_{2} \mathrm{SiO}_{4}$ based on two cations for four oxygen atoms. According to the point analysis results and the comparison of the $\left(M g / F e^{2}+M g\right)$ and $\left(F e^{2} /\left(F e^{2}+M g\right)\right.$ charts, the chemical composition of the olivine from intermediate and basic rocks of the region corresponds to the hyalosiderite to hortonlite range (Fig. 5) (Deer et al. 1992). 
Periódico do Núcleo de Estudos e Pesquisas sobre Gênero e Direito

Centro de Ciências Jurídicas - Universidade Federal da Paraíba

V. 8 - $\mathrm{N}^{\circ} 03$ - Ano 2019

ISSN | 2179-7137 | http://periodicos.ufpb.br/ojs2/index.php/ged/index

a

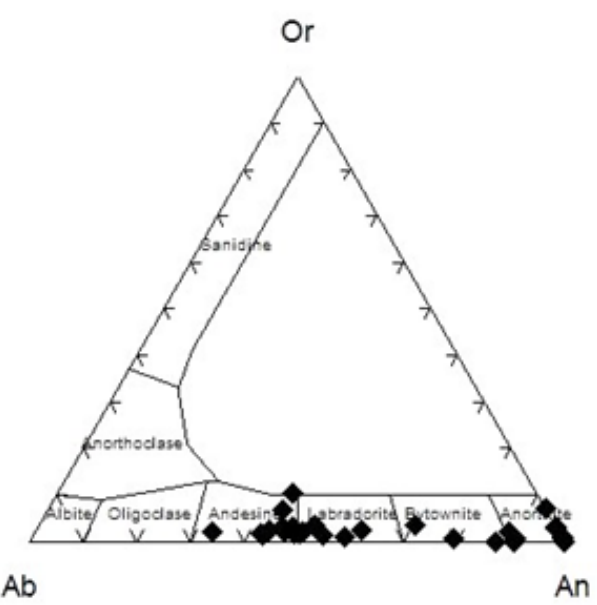

$A b$

$\mathrm{c}$ b

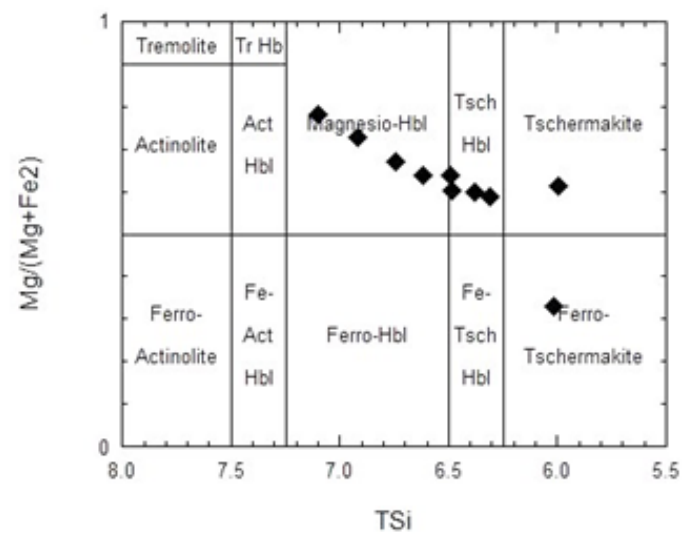

d
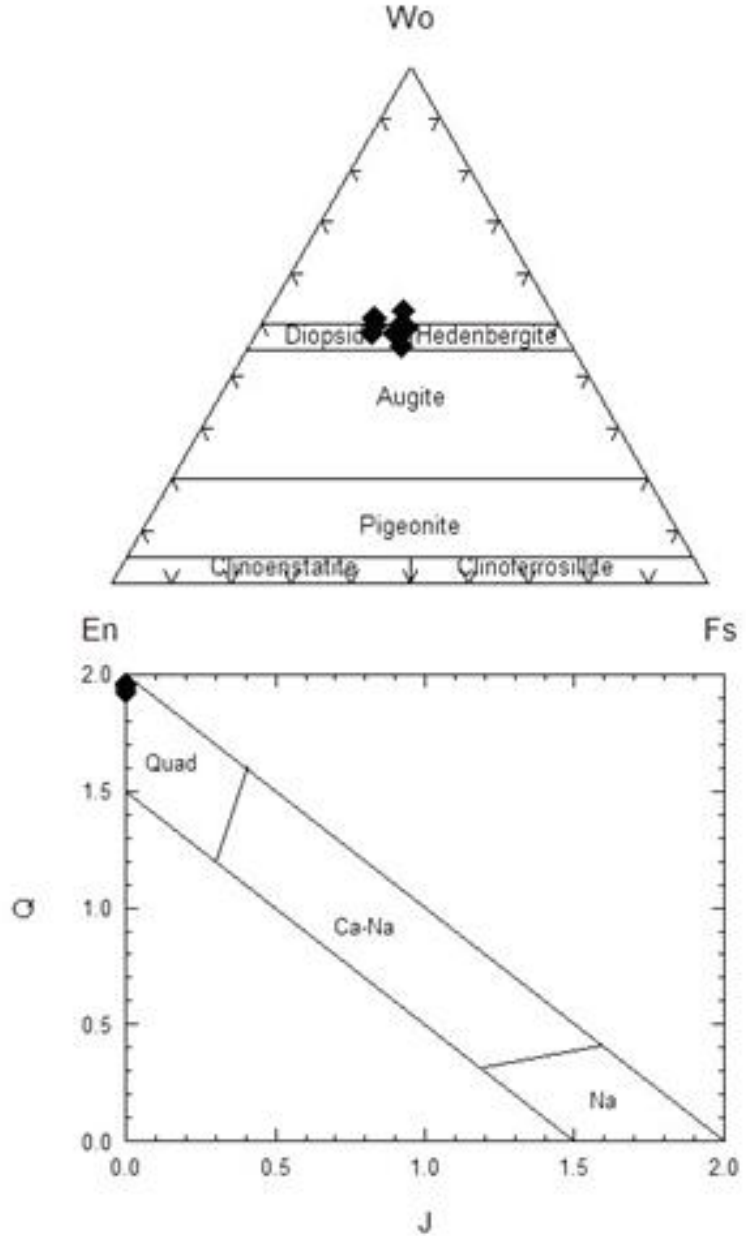


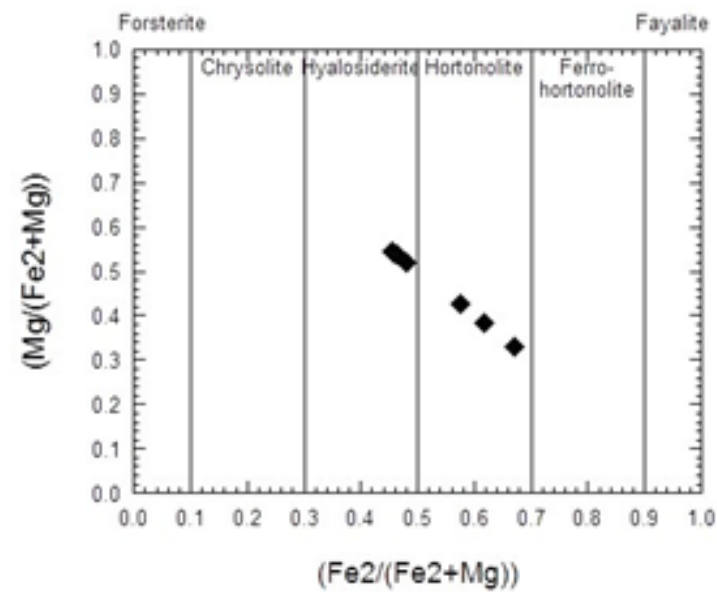

Figure5. a) classification of feldspars based on chemical composition (Deer et al., 1992); b) classification of amphiboles based on the method proposed by Leake et al., 1997; c) $Q-J$ diagram and the standing of pyroxenes in the Quad region (Morimoto et al., 1988); d) classification of the studied pyroxenes and their placement in the hybrid diopside range (Morimoto et al., 1988); e) olivine type identification diagram based on its chemical composition (Deer et al., 1992).

\section{Geochemistry}

An advantageous method of petrogenetic interpretation of intrusive rocks is to make use of geochemical data and investigate the changes and ratios of main and rare elements in the rock mass. As evident from Table 1, the $\mathrm{SiO}_{2}$ content in the studied intrusive masses is variable between 44.18 and $69.09 \%$. According to the Total Alkali Silica (TAS) diagram (Cox et al. 1979), the studied granitoid rocks belong to the granite (subalkaline) to granodiorite range, dioritic rocks to the diorite range (subalkaline), monzodiorite (enclave), syenite (alkaline), and gabbroids to the subalkaline gabbro range. Meanwhile, all data are located in the vicinity of the alkaline-subalkaline boundary as all samples belong to the subalkaline range, except for syenite. According to the $K$ classification diagram for igneous rocks (Peccerillo and Taylor, 1976), the intrusive rocks in the study region generally belong to the potassium-rich, calc-alkaline series. Based on rock rating by alumin saturation (Shand, 1943), if $A / C N K<1$, the granite is I-Type and from the weak meta-alumin to peralumin range, whereas for a molar ratio of over 1.1, the granite is S-Type and peralumin (Frost, 2001). As evident from 
the diagram, the specimens belong to the meta-alumin-per-alumin domain, and the molar ratio is below 1.1 in all samples, indicating the I-type nature of the studied granites. By comparison of the field specifications, mineralogy, geochemistry, and comparative charts identifying the source of granites (Chappell and White, 2001; Mobashergarmi et al., 2018), it was revealed that the rocks in the study region match the specifications of the $I$ Type granite. A comparison of the mineralogy and geochemistry of the granites in the region with the orogeny granitoids (IAG, CAG, CCG, POG) presented by Maniar and Piccoli, 1989, reveals that samples from the study region show the highest consistency with the Continental Collision Granitoids (CCG), Island Arc Granitoids (IAG), and Continental Arc Granitoids (CAG). However, given the mineralogy and geology of the region, these granitoids are precisely of the $C A G$ group, in other words, continental subduction zone. Subduction zone, as regards the tectonic setting of these rocks, refers to the subduction of the oceanic crust, Neotethys, under the central Iranian continental crust, which results in a protrusion following the collision of the

\section{5}

Arabian and Iranian plates. One purpose of rare elements in solving geological problems is to use them to identify the source and genesis of rock systems. The ratio of these elements does not change during metasomatism and alteration. The normalized multi-element diagram (spider diagram) is one example of diagrams used for this purpose. The chondrite-normalized REE multielement diagram (Boynton, 1984), and the primitive-mantle-normalized multielement diagram (Wood et al., 1979) were plotted for the studied samples. According to these plots, all samples exhibit saturation with chondrites and the primitive mantle, which is higher for Light Rare Earth Elements (LREE) than Heavy Rare Earth Elements (HREE) and features a decreasing slope from left to right. The HREE change at a slow rate, but the LREE exhibit higher slopes, except for basic rocks for which the slope is lower than others. Overall, the changes are not steep among the REE, indicating the uniform composition of the parent rock. The REE have little solubility and remain relatively undisturbed by weathering, low-grade metasomatism, and hydrothermal alteration. Further, as evident, higher LREE saturation than HREE is 
characteristic to calc-alkaline magma series (Fig. 6).

Feldspars remain in control of the $E u$ anomaly, since $E u^{2+}$ is compatible in plagioclases and the separation of these minerals by partial melting or crystal fractionation creates a negative $E u$ anomaly in the melt. Crystallization of such minerals as clinopyroxene, sphene, and garnet induces a positive $E u$ anomaly in the residual melt. Further, the lack of this anomaly is suggestive of the insignificant role of calcic plagioclase crystallization in magma evolution. Besides the above, the positive or negative sign of the $E u$ anomaly depends on the oxygen fugacity and the temperature. Therefore, oxygen fugacity also controls the $E u$ anomaly and promotes a positive $E u$ anomaly that is similar to the other REE under $E u$ oxidation conditions. The positive $E u$ anomaly in these samples can be the result of plagioclase phenocrysts in these rocks and, additionally, the high contents of $\mathrm{La}$ and $\mathrm{Ce}$ (from light, incompatible elements) can be attributed to the presence of apatite minerals (Rollinson, 1993).

The primitive-mantlenormalized multi-element spider diagram in Fig. 1.g shows negative $Z r$, $T a$, and $T i$ anomalies, as well as a positive $K$ anomaly. Overall, the decreasing slope of the diagram from left to right is suggestive of magma fractionation, and all elements exhibit saturation in chondrites and the primitive mantle, which is consistent with subduction patterns (Rollinson, 1993). a

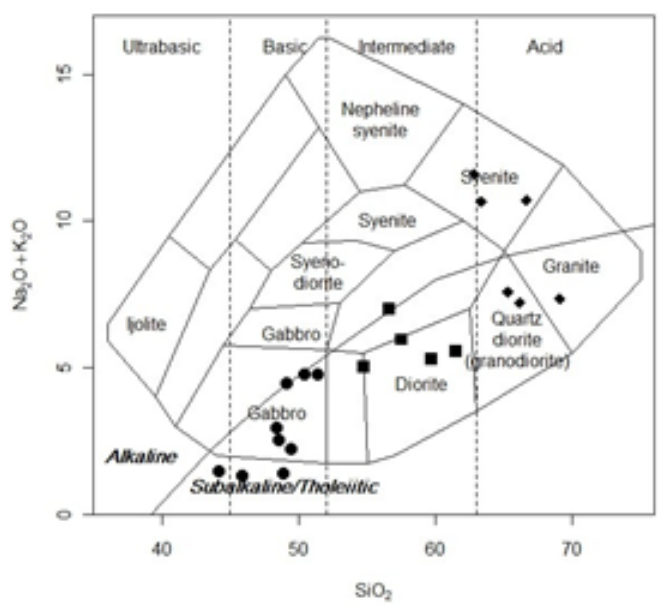

\section{Legend}

- Acid rocks (e.g., granite; syenite)

Intermediate rocks (e.g., diorite)

Basic rocks (e.g., gabbro) $\mathrm{b}$

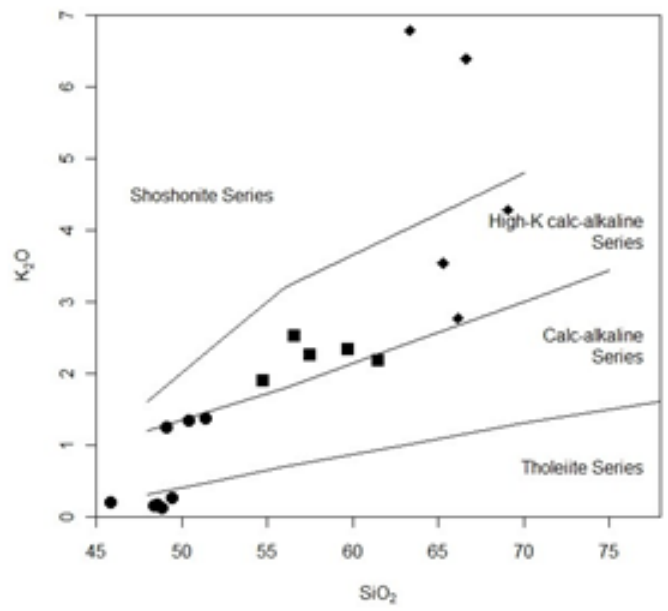



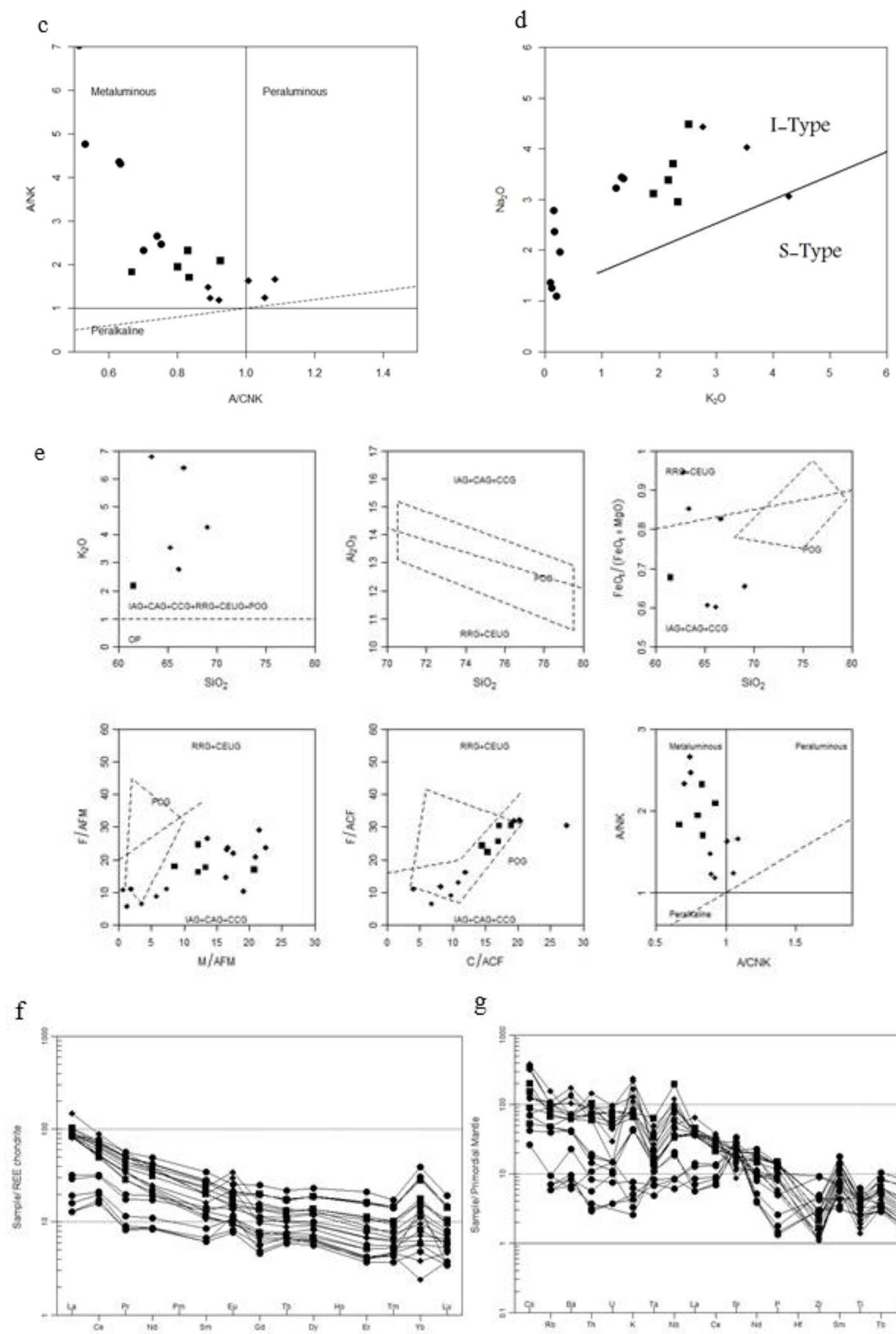

g

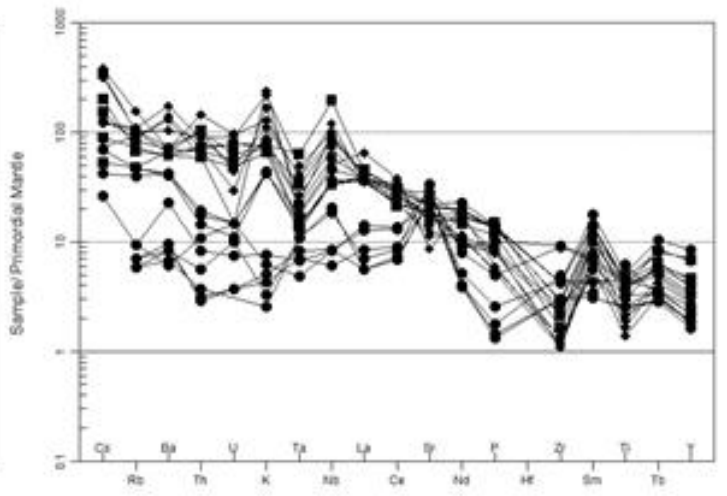


Figure 6. a) plutonic rock rating using the TAS (Cox et al., 1979); b) $k$-classification diagram for igneous rocks (Peccerillo and Taylor, 1976); c) alumin saturation for samples from the intrusive rock based on the $\mathrm{A} / \mathrm{CNK}-\mathrm{A} / \mathrm{NK}$ diagram (Shand, 1943); d) $\mathrm{Na}_{2} \mathrm{O}$ against $\mathrm{K}_{2} \mathrm{O}$ (Chappell and White, 2001); e) tectonic setting discrimination diagrams (Maniar and Piccoli, 1989); f) chondrite-normalized REE diagram (Boynton, 1984); g) primitive-mantle-normalized spider diagram (Wood et al., 1979).

\section{Conclusion}

Petrographical and field studies show the intrusive rocks south of Naqadeh to be of acidic, intermediate, and basic types. These components come with various petrologies including syenogranites, monzogranite, granodiorites, quartz diorites, quartz monzonites, quartz monzonite, quartz syenite, and olivine gabbro-in order of abundance. Minerals forming intrusive rocks include alkali feldspar, quartz, plagioclase (andesine to anorthite), amphibole (magnesiohornblende tschermakite hornblende), pyroxene (diopside), olivine (hyalosiderite to hortonlite), apatite, titanite, zircon, muscovite, and opaque minerals. The chemical analysis of the local granitoids revealed their potassium-rich, calcalkaline nature, falling to the metaalumin to per-alumin range in terms of saturation. However, based on the tectonomagmatic diagrams, the studied granitoid rocks show the highest consistency with the $C A G$ group, or in other words, the continental-subduction zone. Subduction zone, as regards the tectonic setting of these rocks, refers to the subduction of the oceanic crust, Neotethys, under the central Iranian continental crust, which results in a protrusion following the collision of the Arabian and Iranian plates (Alavi, 2008; Shahabpour, 2004; Hassanzadeh et al., 2007). A comparison of field observations, mineralogy, geochemistry, and diagrams illustrating the sources of granites showed that the intrusive rocks in the study region are Type-I granite, and that the magmatism is a result of the subduction of the oceanic crust under the continental crust (Sheikholeslami et al., 2008). Based on the above discussions, the considerable role of the lower crust is evident as regards the source of granite (acidic) rocks in the region. As a result of the intrusion of basic magma from the upper mantle, crust materials are partially melted and digested, creating an intermediate magma which is rich in lithophile elements and, compared to the 


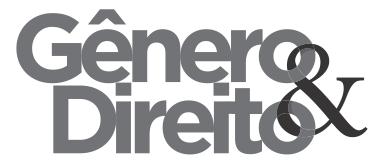

Periódico do Núcleo de Estudos e Pesquisas sobre Gênero e Direito

Centro de Ciências Jurídicas - Universidade Federal da Paraíba

V. 8 - $\mathrm{N}^{\circ} 03$ - Ano 2019

ISSN | 2179-7137 | http://periodicos.ufpb.br/ojs2/index.php/ged/index

initial magma, has a higher capacity for

producing large volumes of granite due to incremental crystallization. Therefore, according to spider diagrams, no depletion of any element was observed. Moreover, a negative slope was observed in general, which is consistent with the subduction patterns.

\section{Acknowledgments}

Authors would like to express their gratitude to the Higher Education Office of the North Tehran Islamic Azad University.

\section{Appendices}

Table 1. XRF analysis results for samples taken from the different parts of the study region $(w t . \%)$

\begin{tabular}{|c|c|c|c|c|c|c|c|c|c|c|c|c|c|c|}
\hline $\begin{array}{c}\text { Sam } \\
\text { ple }\end{array}$ & $\begin{array}{l}\mathrm{Si} \\
\mathrm{O}_{2}\end{array}$ & $\begin{array}{l}\mathrm{Al}_{2} \\
\mathrm{O}_{3}\end{array}$ & $\begin{array}{c}\mathrm{Ba} \\
\mathrm{O}\end{array}$ & $\begin{array}{c}\mathrm{Ca} \\
\mathrm{O}\end{array}$ & $\begin{array}{c}\mathrm{Fe}_{2} \\
\mathrm{O}_{3}\end{array}$ & $\begin{array}{c}\mathrm{K}_{2} \\
\mathrm{O}\end{array}$ & $\begin{array}{c}\mathrm{Mg} \\
\mathrm{O}\end{array}$ & $\begin{array}{c}\mathrm{Mn} \\
\mathrm{O}\end{array}$ & $\begin{array}{c}\mathrm{Na}_{2} \\
\mathrm{O}\end{array}$ & $\begin{array}{l}\mathrm{P}_{2} \\
\mathrm{O}_{5}\end{array}$ & $\begin{array}{c}\mathrm{S} \\
\mathrm{O}_{3}\end{array}$ & $\begin{array}{c}\mathrm{Ti} \\
\mathrm{O}_{2}\end{array}$ & $\begin{array}{c}\mathrm{L} \\
\mathrm{OI}\end{array}$ & $\begin{array}{c}\text { Tot } \\
\text { al }\end{array}$ \\
\hline $\mathrm{DL}^{*}$ & $\begin{array}{c}0.0 \\
5\end{array}$ & $\begin{array}{l}0.0 \\
5\end{array}$ & $\begin{array}{l}0.0 \\
5\end{array}$ & $\begin{array}{l}0.0 \\
5\end{array}$ & $\begin{array}{l}0.0 \\
5\end{array}$ & $\begin{array}{l}0 . \\
05\end{array}$ & $\begin{array}{l}0.0 \\
5\end{array}$ & $\begin{array}{l}0.0 \\
5\end{array}$ & $\begin{array}{l}0.0 \\
5\end{array}$ & $\begin{array}{l}0.0 \\
5\end{array}$ & $\begin{array}{l}0 . \\
05\end{array}$ & $\begin{array}{l}0.0 \\
5\end{array}$ & $\begin{array}{l}0 . \\
05\end{array}$ & \\
\hline A39 & $\begin{array}{c}69 . \\
09\end{array}$ & $\begin{array}{l}16 . \\
05\end{array}$ & $\begin{array}{c}0.0 \\
7\end{array}$ & $\begin{array}{c}2.8 \\
2\end{array}$ & $\begin{array}{c}1.9 \\
2\end{array}$ & $\begin{array}{l}4 . \\
28\end{array}$ & $\begin{array}{c}0.9 \\
1\end{array}$ & $\begin{array}{c}0.0 \\
6\end{array}$ & $\begin{array}{c}3.0 \\
6\end{array}$ & $\begin{array}{c}0.1 \\
4\end{array}$ & $<$ & $\begin{array}{c}0.3 \\
3\end{array}$ & $\begin{array}{l}1 . \\
27\end{array}$ & 100 \\
\hline A61 & $\begin{array}{c}66 . \\
16\end{array}$ & $\begin{array}{l}15 . \\
22\end{array}$ & $\begin{array}{c}0.0 \\
6\end{array}$ & $\begin{array}{c}3.7 \\
4\end{array}$ & $\begin{array}{c}3.4 \\
2\end{array}$ & $\begin{array}{l}2 . \\
77\end{array}$ & $\begin{array}{c}2.0 \\
3\end{array}$ & $\begin{array}{c}0.0 \\
7\end{array}$ & $\begin{array}{c}4.4 \\
4\end{array}$ & 0.2 & $<$ & $\begin{array}{c}0.5 \\
4\end{array}$ & $\begin{array}{l}1 . \\
34\end{array}$ & $\begin{array}{c}99 . \\
99\end{array}$ \\
\hline A81 & $\begin{array}{c}56 . \\
59\end{array}$ & $\begin{array}{r}17 . \\
15\end{array}$ & $\begin{array}{c}0.0 \\
6\end{array}$ & $\begin{array}{c}5.7 \\
3\end{array}$ & $\begin{array}{c}6.0 \\
7\end{array}$ & $\begin{array}{l}2 . \\
52\end{array}$ & $\begin{array}{c}4.1 \\
2\end{array}$ & $\begin{array}{c}0.1 \\
6\end{array}$ & $\begin{array}{c}4.4 \\
8\end{array}$ & $\begin{array}{c}0.3 \\
1\end{array}$ & $<$ & 0.9 & $\begin{array}{l}. \\
91\end{array}$ & 100 \\
\hline $\begin{array}{c}\mathrm{A} 10 \\
5\end{array}$ & $\begin{array}{l}65 . \\
28\end{array}$ & $\begin{array}{l}17 . \\
03\end{array}$ & $\begin{array}{c}0.0 \\
9\end{array}$ & $\begin{array}{c}3.5 \\
4\end{array}$ & $\begin{array}{c}2.8 \\
7\end{array}$ & $\begin{array}{l}3 . \\
54\end{array}$ & $\begin{array}{c}1.6 \\
7\end{array}$ & $\begin{array}{c}0.0 \\
7\end{array}$ & $\begin{array}{c}4.0 \\
3\end{array}$ & $\begin{array}{c}0.1 \\
7\end{array}$ & $<$ & $\begin{array}{c}0.4 \\
9\end{array}$ & $\begin{array}{l}1 . \\
22\end{array}$ & 100 \\
\hline $\begin{array}{c}\text { A11 } \\
5\end{array}$ & $\begin{array}{l}62 . \\
83\end{array}$ & $\begin{array}{r}18 . \\
17\end{array}$ & $\begin{array}{c}0.1 \\
1\end{array}$ & $\begin{array}{c}1.4 \\
1\end{array}$ & $\begin{array}{c}4.0 \\
7\end{array}$ & $\begin{array}{l}7 . \\
87\end{array}$ & $\begin{array}{c}0.2 \\
1\end{array}$ & $\begin{array}{c}0.0 \\
8\end{array}$ & $\begin{array}{c}3.7 \\
3\end{array}$ & $\begin{array}{c}0.1 \\
1\end{array}$ & $<$ & $\begin{array}{c}0.4 \\
1\end{array}$ & $\begin{array}{l}0 . \\
81\end{array}$ & $\begin{array}{c}99 . \\
81\end{array}$ \\
\hline $\begin{array}{c}\text { A13 } \\
7\end{array}$ & $\begin{array}{c}44 . \\
18\end{array}$ & $\begin{array}{l}16 . \\
44\end{array}$ & $<$ & $\begin{array}{c}16 . \\
3\end{array}$ & $\begin{array}{l}11 . \\
62\end{array}$ & $\begin{array}{c}0 . \\
09\end{array}$ & $\begin{array}{c}7.7 \\
5\end{array}$ & $\begin{array}{c}0.1 \\
4\end{array}$ & $\begin{array}{c}1.3 \\
6\end{array}$ & $<$ & $<$ & $\begin{array}{c}1.6 \\
7\end{array}$ & $\begin{array}{c}0 . \\
38\end{array}$ & $\begin{array}{l}99 . \\
93\end{array}$ \\
\hline $\begin{array}{c}\text { A14 } \\
1\end{array}$ & $\begin{array}{l}66 . \\
64\end{array}$ & $\begin{array}{c}16 . \\
54\end{array}$ & $\begin{array}{c}0.1 \\
1\end{array}$ & $\begin{array}{c}2.1 \\
5\end{array}$ & $\begin{array}{c}1.9 \\
1\end{array}$ & $\begin{array}{c}6 . \\
39\end{array}$ & $\begin{array}{c}0.3 \\
6\end{array}$ & $<$ & $\begin{array}{c}4.3 \\
2\end{array}$ & 0.1 & $<$ & $\begin{array}{c}0.3 \\
7\end{array}$ & $\begin{array}{c}1 . \\
1\end{array}$ & $\begin{array}{c}99 . \\
99\end{array}$ \\
\hline $\begin{array}{c}\text { A16 } \\
2\end{array}$ & $\begin{array}{l}63 . \\
35\end{array}$ & $\begin{array}{l}16 . \\
86\end{array}$ & $\begin{array}{c}0.1 \\
4\end{array}$ & $\begin{array}{c}2.8 \\
0\end{array}$ & $\begin{array}{c}3.8 \\
7\end{array}$ & $\begin{array}{c}6 . \\
79\end{array}$ & 0.6 & $\begin{array}{c}0.0 \\
5\end{array}$ & $\begin{array}{c}3.8 \\
7\end{array}$ & $\begin{array}{c}0.1 \\
5\end{array}$ & $<$ & $\begin{array}{c}0.6 \\
3\end{array}$ & $\begin{array}{c}0 . \\
81\end{array}$ & $\begin{array}{c}99 . \\
92\end{array}$ \\
\hline $\begin{array}{c}\text { A16 } \\
3\end{array}$ & $\begin{array}{l}57 . \\
49\end{array}$ & $\begin{array}{l}16 . \\
56\end{array}$ & $\begin{array}{c}0.0 \\
5\end{array}$ & $\begin{array}{c}6.6 \\
8\end{array}$ & $\begin{array}{c}6.3 \\
6\end{array}$ & $\begin{array}{l}2 . \\
25\end{array}$ & $\begin{array}{c}4.3 \\
4\end{array}$ & $\begin{array}{c}0.1 \\
1\end{array}$ & 3.7 & $\begin{array}{c}0.2 \\
8\end{array}$ & $<$ & $\begin{array}{c}0.9 \\
5\end{array}$ & 1. & $\begin{array}{c}99 . \\
99\end{array}$ \\
\hline
\end{tabular}


Periódico do Núcleo de Estudos e Pesquisas sobre Gênero e Direito Centro de Ciências Jurídicas - Universidade Federal da Paraíba V. 8 - $\mathrm{N}^{\circ} 03$ - Ano 2019 ISSN | 2179-7137 | http://periodicos.ufpb.br/ojs2/index.php/ged/index

\begin{tabular}{|c|c|c|c|c|c|c|c|c|c|c|c|c|c|c|}
\hline $\begin{array}{c}\text { A17 } \\
4\end{array}$ & $\begin{array}{l}59 . \\
73\end{array}$ & $\begin{array}{l}13 . \\
46\end{array}$ & $\begin{array}{c}0.0 \\
6\end{array}$ & $\begin{array}{c}7.0 \\
3\end{array}$ & $\begin{array}{c}5.6 \\
5\end{array}$ & $\begin{array}{c}2 . \\
33\end{array}$ & $\begin{array}{c}6.2 \\
4\end{array}$ & $\begin{array}{c}0.1 \\
3\end{array}$ & $\begin{array}{c}2.9 \\
5\end{array}$ & $\begin{array}{c}0.1 \\
8\end{array}$ & $\begin{array}{l}0 . \\
05\end{array}$ & $\begin{array}{c}0.7 \\
1\end{array}$ & $\begin{array}{c}1 . \\
48\end{array}$ & 100 \\
\hline $\begin{array}{c}\text { A17 } \\
7\end{array}$ & $\begin{array}{l}48 . \\
84\end{array}$ & $\begin{array}{l}20 . \\
95\end{array}$ & $<$ & $\begin{array}{l}17 . \\
26\end{array}$ & $\begin{array}{c}3.6 \\
3\end{array}$ & $\begin{array}{c}0 . \\
12\end{array}$ & $\begin{array}{c}6.0 \\
4\end{array}$ & $\begin{array}{c}0.0 \\
6\end{array}$ & $\begin{array}{c}1.2 \\
6\end{array}$ & $<$ & $\begin{array}{l}0 . \\
18\end{array}$ & $\begin{array}{c}0.5 \\
7\end{array}$ & $\begin{array}{l}1 . \\
04\end{array}$ & $\begin{array}{c}99 . \\
95\end{array}$ \\
\hline $\begin{array}{c}\text { A19 } \\
3\end{array}$ & $\begin{array}{c}49 . \\
40\end{array}$ & $\begin{array}{l}16 . \\
73\end{array}$ & $<$ & $\begin{array}{l}15 . \\
42\end{array}$ & $\begin{array}{c}7.6 \\
4\end{array}$ & $\begin{array}{l}0 . \\
26\end{array}$ & $\begin{array}{c}5.5 \\
2\end{array}$ & $\begin{array}{c}0.1 \\
3\end{array}$ & $\begin{array}{c}1.9 \\
6\end{array}$ & $\begin{array}{c}0.1 \\
1\end{array}$ & $<$ & $\begin{array}{c}1.5 \\
4\end{array}$ & $\begin{array}{l}1 . \\
29\end{array}$ & 100 \\
\hline $\begin{array}{c}\text { A20 } \\
3\end{array}$ & $\begin{array}{l}45 . \\
85\end{array}$ & $\begin{array}{l}15 . \\
22\end{array}$ & $<$ & $\begin{array}{l}19 . \\
55\end{array}$ & 8 & $\begin{array}{l}0 . \\
2\end{array}$ & $\begin{array}{c}6.8 \\
9\end{array}$ & $\begin{array}{c}0.1 \\
3\end{array}$ & 1.1 & $\begin{array}{c}0.2 \\
4\end{array}$ & $<$ & $\begin{array}{c}1.5 \\
7\end{array}$ & $\begin{array}{l}1 . \\
25\end{array}$ & 100 \\
\hline $\begin{array}{c}\mathrm{A} 22 \\
5\end{array}$ & $\begin{array}{c}49 . \\
13\end{array}$ & $\begin{array}{l}17 . \\
66\end{array}$ & $<$ & $\begin{array}{c}9.4 \\
4\end{array}$ & $\begin{array}{l}10 . \\
89\end{array}$ & $\begin{array}{l}1 . \\
25\end{array}$ & 5 & $\begin{array}{c}0.2 \\
1\end{array}$ & $\begin{array}{c}3.2 \\
2\end{array}$ & $\begin{array}{c}0.2 \\
9\end{array}$ & $\begin{array}{l}0 . \\
05\end{array}$ & $\begin{array}{c}1.1 \\
5\end{array}$ & $\begin{array}{l}1 . \\
71\end{array}$ & 100 \\
\hline $\begin{array}{c}\mathrm{A} 23 \\
0\end{array}$ & $\begin{array}{l}54 . \\
73\end{array}$ & $\begin{array}{r}16 . \\
65\end{array}$ & $\begin{array}{c}0.0 \\
5\end{array}$ & $\begin{array}{c}7.0 \\
7\end{array}$ & $\begin{array}{c}9.3 \\
7\end{array}$ & $\begin{array}{l}1 . \\
9\end{array}$ & $\begin{array}{c}4.1 \\
7\end{array}$ & $\begin{array}{c}0.1 \\
9\end{array}$ & $\begin{array}{c}3.1 \\
1\end{array}$ & $\begin{array}{c}0.2 \\
7\end{array}$ & $<$ & $\begin{array}{c}1.0 \\
1\end{array}$ & $\begin{array}{c}1 . \\
48\end{array}$ & 100 \\
\hline $\begin{array}{c}\text { A23 } \\
2 \mathrm{~A}\end{array}$ & $\begin{array}{l}51 . \\
38\end{array}$ & $\begin{array}{l}16 . \\
54\end{array}$ & $<$ & $\begin{array}{c}9.0 \\
6\end{array}$ & $\begin{array}{c}9.3 \\
6\end{array}$ & $\begin{array}{l}1 . \\
37\end{array}$ & $\begin{array}{c}5.9 \\
5\end{array}$ & $\begin{array}{c}0.1 \\
9\end{array}$ & $\begin{array}{c}3.4 \\
1\end{array}$ & $\begin{array}{c}0.2 \\
7\end{array}$ & $<$ & $\begin{array}{c}0.8 \\
9\end{array}$ & $\begin{array}{l}1 . \\
57\end{array}$ & $\begin{array}{c}99 . \\
99\end{array}$ \\
\hline $\begin{array}{c}\text { A23 } \\
2 B\end{array}$ & $\begin{array}{c}50 . \\
41\end{array}$ & $\begin{array}{l}17 . \\
51\end{array}$ & $<$ & $\begin{array}{c}8.8 \\
7\end{array}$ & $\begin{array}{c}9.4 \\
2\end{array}$ & $\begin{array}{l}1 . \\
34\end{array}$ & $\begin{array}{c}6.1 \\
1\end{array}$ & 0.2 & $\begin{array}{c}3.4 \\
4\end{array}$ & $\begin{array}{c}0.2 \\
7\end{array}$ & $<$ & $\begin{array}{c}0.8 \\
7\end{array}$ & $\begin{array}{l}1 . \\
56\end{array}$ & 100 \\
\hline $\begin{array}{c}\text { A23 } \\
6\end{array}$ & $\begin{array}{l}61 . \\
48\end{array}$ & $\begin{array}{l}16 . \\
51\end{array}$ & $<$ & $\begin{array}{c}5.4 \\
5\end{array}$ & $\begin{array}{c}5.9 \\
4\end{array}$ & $\begin{array}{l}2 . \\
17\end{array}$ & $\begin{array}{c}2.5 \\
6\end{array}$ & $\begin{array}{c}0.1 \\
2\end{array}$ & $\begin{array}{c}3.3 \\
8\end{array}$ & $\begin{array}{c}0.2 \\
3\end{array}$ & $<$ & $\begin{array}{c}0.7 \\
3\end{array}$ & $\begin{array}{l}1 . \\
39\end{array}$ & $\begin{array}{c}99 . \\
96\end{array}$ \\
\hline $\begin{array}{c}\text { A23 } \\
7\end{array}$ & $\begin{array}{c}48 . \\
57\end{array}$ & $\begin{array}{l}17 . \\
77\end{array}$ & $<$ & $\begin{array}{l}13 . \\
25\end{array}$ & $\begin{array}{c}8.0 \\
8\end{array}$ & $\begin{array}{c}0 . \\
16\end{array}$ & 7.3 & $\begin{array}{c}0.1 \\
4\end{array}$ & $\begin{array}{c}2.3 \\
7\end{array}$ & $<$ & $\begin{array}{l}0 . \\
37\end{array}$ & $\begin{array}{c}1.0 \\
6\end{array}$ & $\begin{array}{c}0 . \\
93\end{array}$ & 100 \\
\hline $\begin{array}{c}\mathrm{A} 24 \\
6\end{array}$ & $\begin{array}{c}48 . \\
40\end{array}$ & $\begin{array}{l}20 . \\
47\end{array}$ & $<$ & $\begin{array}{l}15 . \\
13\end{array}$ & $\begin{array}{c}5.4 \\
1\end{array}$ & $\begin{array}{c}0 . \\
15\end{array}$ & $\begin{array}{c}5.5 \\
4\end{array}$ & $\begin{array}{c}0.1 \\
1\end{array}$ & $\begin{array}{c}2.7 \\
9\end{array}$ & $<$ & $\begin{array}{l}0 . \\
21\end{array}$ & $\begin{array}{c}0.5 \\
9\end{array}$ & $\begin{array}{l}1 . \\
2\end{array}$ & 100 \\
\hline
\end{tabular}

Table 2. ICP-MS analysis results for samples taken from the different parts of the study region $(\mathrm{ppm})$

\begin{tabular}{|c|c|c|c|c|c|c|c|c|c|c|c|c|c|c|}
\hline $\begin{array}{c}\text { Samp } \\
\text { le }\end{array}$ & $\mathrm{Ag}$ & $\mathrm{Al}$ & As & $\mathrm{Ba}$ & $\begin{array}{l}\text { B } \\
\text { e }\end{array}$ & $\mathrm{Bi}$ & $\mathrm{Ca}$ & $\mathrm{Cd}$ & $\begin{array}{l}\mathrm{C} \\
\mathrm{e}\end{array}$ & Co & $\mathrm{Cr}$ & Cs & $\mathrm{Cu}$ & Dy \\
\hline $\mathrm{DL}^{*}$ & 0.1 & 100 & 0.1 & 1 & $\begin{array}{l}0 . \\
2\end{array}$ & $\begin{array}{c}0 . \\
1\end{array}$ & 100 & 0.1 & $\begin{array}{c}0 . \\
5\end{array}$ & 1 & 1 & 0.5 & 1 & $\begin{array}{c}0.0 \\
2\end{array}$ \\
\hline A39 & $\begin{array}{c}<0 . \\
1\end{array}$ & $\begin{array}{c}747 \\
42\end{array}$ & 0.8 & 537 & $\begin{array}{l}2 . \\
5\end{array}$ & $\begin{array}{l}0 . \\
2\end{array}$ & $\begin{array}{c}171 \\
28\end{array}$ & $\begin{array}{c}<0 . \\
1\end{array}$ & $\begin{array}{l}4 \\
2\end{array}$ & 4.8 & 12 & 7.3 & 9 & $\begin{array}{c}1.8 \\
8\end{array}$ \\
\hline A61 & $\begin{array}{l}<0 . \\
1\end{array}$ & $\begin{array}{c}766 \\
00\end{array}$ & 0.9 & 557 & $\begin{array}{l}1 . \\
8\end{array}$ & $\begin{array}{l}0 . \\
2\end{array}$ & $\begin{array}{c}269 \\
05\end{array}$ & $\begin{array}{c}<0 . \\
1\end{array}$ & $\begin{array}{l}4 \\
2\end{array}$ & $\begin{array}{c}10 . \\
3\end{array}$ & 14 & 6 & 11 & $\begin{array}{c}2.3 \\
4\end{array}$ \\
\hline A81 & $\begin{array}{l}<0 . \\
1\end{array}$ & $\begin{array}{c}782 \\
93\end{array}$ & 0.4 & 491 & $\begin{array}{l}2 . \\
4\end{array}$ & $\begin{array}{l}0 . \\
2\end{array}$ & $\begin{array}{c}370 \\
07\end{array}$ & 0.1 & $\begin{array}{l}6 \\
0\end{array}$ & 18 & 31 & 6.5 & 57 & $\begin{array}{c}4.0 \\
1\end{array}$ \\
\hline
\end{tabular}




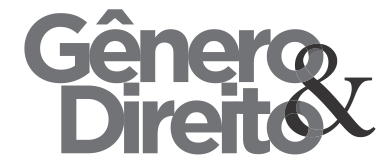

Periódico do Núcleo de Estudos e Pesquisas sobre Gênero e Direito Centro de Ciências Jurídicas - Universidade Federal da Paraíba V. 8 - $\mathrm{N}^{\circ} 03$ - Ano 2019 ISSN | 2179-7137 | http://periodicos.ufpb.br/ojs2/index.php/ged/index

\begin{tabular}{|c|c|c|c|c|c|c|c|c|c|c|c|c|c|c|}
\hline A105 & $\begin{array}{l}<0 . \\
1\end{array}$ & $\begin{array}{c}636 \\
87\end{array}$ & 0.6 & 781 & $\begin{array}{l}1 . \\
7\end{array}$ & $\begin{array}{c}0 . \\
1\end{array}$ & $\begin{array}{c}216 \\
43\end{array}$ & $\begin{array}{c}<0 . \\
1\end{array}$ & $\begin{array}{l}4 \\
2\end{array}$ & 8 & 12 & 2.4 & 6 & $\begin{array}{c}2.0 \\
4\end{array}$ \\
\hline A115 & $\begin{array}{l}<0 . \\
1\end{array}$ & $\begin{array}{c}826 \\
60\end{array}$ & 0.3 & 987 & $\begin{array}{l}1 . \\
2\end{array}$ & $\begin{array}{c}0 . \\
1\end{array}$ & $\begin{array}{c}977 \\
4\end{array}$ & $\begin{array}{c}<0 . \\
1\end{array}$ & $\begin{array}{l}7 \\
2\end{array}$ & 2.6 & 5 & 1.4 & 3 & $\begin{array}{c}3.0 \\
7\end{array}$ \\
\hline A137 & 0.1 & $\begin{array}{c}881 \\
41\end{array}$ & $\begin{array}{c}<0 . \\
1\end{array}$ & 51 & $\begin{array}{l}0 . \\
3\end{array}$ & $\begin{array}{l}0 . \\
2\end{array}$ & $\begin{array}{c}>10 \\
\%\end{array}$ & 0.1 & $\begin{array}{l}1 \\
3\end{array}$ & $\begin{array}{c}55 . \\
6\end{array}$ & 36 & $\begin{array}{c}<0 . \\
5\end{array}$ & $\begin{array}{c}42 \\
2\end{array}$ & $\begin{array}{c}2.1 \\
1\end{array}$ \\
\hline A141 & $\begin{array}{l}<0 . \\
1\end{array}$ & $\begin{array}{c}802 \\
64\end{array}$ & 2 & $\begin{array}{c}105 \\
5\end{array}$ & $\begin{array}{l}1 . \\
8\end{array}$ & $\begin{array}{c}0 . \\
1\end{array}$ & $\begin{array}{c}148 \\
31\end{array}$ & $\begin{array}{c}<0 . \\
1\end{array}$ & $\begin{array}{l}4 \\
3\end{array}$ & 2.3 & 8 & 2.5 & 8 & $\begin{array}{c}2.9 \\
4\end{array}$ \\
\hline A162 & $\begin{array}{l}<0 . \\
1\end{array}$ & $\begin{array}{c}837 \\
60\end{array}$ & 0.6 & $\begin{array}{c}130 \\
9\end{array}$ & $\begin{array}{l}1 . \\
4\end{array}$ & $\begin{array}{l}0 . \\
2\end{array}$ & $\begin{array}{c}195 \\
90\end{array}$ & $\begin{array}{c}<0 . \\
1\end{array}$ & $\begin{array}{l}5 \\
2\end{array}$ & 3.3 & 11 & 2.3 & 4 & $\begin{array}{c}4.3 \\
1\end{array}$ \\
\hline A163 & $\begin{array}{l}<0 . \\
1\end{array}$ & $\begin{array}{c}902 \\
34\end{array}$ & 2.2 & 463 & $\begin{array}{l}1 . \\
8\end{array}$ & $\begin{array}{l}0 . \\
2\end{array}$ & $\begin{array}{c}470 \\
27\end{array}$ & 0.1 & $\begin{array}{l}5 \\
6\end{array}$ & $\begin{array}{c}20 . \\
5\end{array}$ & 90 & 3.8 & 31 & $\begin{array}{c}4.4 \\
3\end{array}$ \\
\hline A174 & $\begin{array}{l}<0 . \\
1\end{array}$ & $\begin{array}{c}670 \\
86\end{array}$ & $\begin{array}{c}<0 . \\
1\end{array}$ & 509 & $\begin{array}{l}1 . \\
4\end{array}$ & $\begin{array}{l}0 . \\
2\end{array}$ & $\begin{array}{c}483 \\
32\end{array}$ & 0.1 & $\begin{array}{l}4 \\
0\end{array}$ & $\begin{array}{c}22 . \\
2\end{array}$ & $\begin{array}{c}26 \\
1\end{array}$ & 1 & 13 & $\begin{array}{c}2.1 \\
9\end{array}$ \\
\hline A177 & $\begin{array}{l}<0 . \\
1\end{array}$ & $\begin{array}{c}>10 \\
\%\end{array}$ & 2 & 63 & $\begin{array}{c}0 . \\
5\end{array}$ & $\begin{array}{l}0 . \\
2\end{array}$ & $\begin{array}{c}>10 \\
\%\end{array}$ & 0.1 & $\begin{array}{l}1 \\
6\end{array}$ & $\begin{array}{c}21 . \\
1\end{array}$ & 91 & $\begin{array}{c}<0 . \\
5\end{array}$ & $\begin{array}{c}10 \\
1\end{array}$ & 1.8 \\
\hline A193 & $\begin{array}{l}<0 . \\
1\end{array}$ & $\begin{array}{c}890 \\
95\end{array}$ & 1.7 & 171 & $\begin{array}{c}0 . \\
7\end{array}$ & $\begin{array}{c}0 . \\
1\end{array}$ & $\begin{array}{c}>10 \\
\%\end{array}$ & $\begin{array}{c}<0 . \\
1\end{array}$ & $\begin{array}{l}2 \\
5\end{array}$ & $\begin{array}{c}26 . \\
5\end{array}$ & 38 & $\begin{array}{c}<0 . \\
5\end{array}$ & 23 & $\begin{array}{c}3.2 \\
2\end{array}$ \\
\hline A203 & 0.1 & $\begin{array}{c}731 \\
02\end{array}$ & 1 & 46 & $\begin{array}{c}0 . \\
9\end{array}$ & $\begin{array}{l}0 . \\
2\end{array}$ & $\begin{array}{c}>10 \\
\%\end{array}$ & 0.2 & $\begin{array}{l}2 \\
6\end{array}$ & $\begin{array}{c}31 . \\
5\end{array}$ & $\begin{array}{c}17 \\
6\end{array}$ & 0.5 & 44 & $\begin{array}{c}3.8 \\
8\end{array}$ \\
\hline A225 & $\begin{array}{l}<0 . \\
1\end{array}$ & $\begin{array}{c}967 \\
71\end{array}$ & 0.5 & 316 & $\begin{array}{l}1 . \\
5\end{array}$ & $\begin{array}{c}0 . \\
1\end{array}$ & $\begin{array}{c}663 \\
37\end{array}$ & $\begin{array}{c}<0 \\
1\end{array}$ & $\begin{array}{l}6 \\
2\end{array}$ & $\begin{array}{c}28 \\
4\end{array}$ & 36 & 0.8 & 92 & $\begin{array}{c}7.4 \\
8\end{array}$ \\
\hline A230 & $\begin{array}{l}<0 . \\
1\end{array}$ & $\begin{array}{c}905 \\
92\end{array}$ & $\begin{array}{l}<0 . \\
1\end{array}$ & 462 & $\begin{array}{l}1 . \\
5\end{array}$ & $\begin{array}{c}0 . \\
1\end{array}$ & $\begin{array}{c}526 \\
72\end{array}$ & $\begin{array}{c}<0 . \\
1\end{array}$ & $\begin{array}{l}5 \\
3\end{array}$ & $\begin{array}{c}23 . \\
2\end{array}$ & 40 & 1.7 & 12 & $\begin{array}{c}6.0 \\
7\end{array}$ \\
\hline $\begin{array}{c}\mathrm{A} 232 \\
\text { A }\end{array}$ & $\begin{array}{l}<0 . \\
1\end{array}$ & $\begin{array}{c}941 \\
44\end{array}$ & $\begin{array}{l}<0 . \\
1\end{array}$ & 318 & $\begin{array}{l}1 . \\
6\end{array}$ & 1 & $\begin{array}{c}661 \\
82\end{array}$ & $\begin{array}{c}<0 \\
1\end{array}$ & $\begin{array}{l}5 \\
8\end{array}$ & $\begin{array}{c}28 . \\
6\end{array}$ & $\begin{array}{c}12 \\
7\end{array}$ & 1.3 & 57 & $\begin{array}{c}6.1 \\
2\end{array}$ \\
\hline $\begin{array}{c}\mathrm{A} 232 \\
\text { B }\end{array}$ & $\begin{array}{l}<0 . \\
1\end{array}$ & $\begin{array}{c}932 \\
09\end{array}$ & $\begin{array}{l}<0 . \\
1\end{array}$ & 309 & $\begin{array}{l}1 . \\
5\end{array}$ & $\begin{array}{c}0 . \\
1\end{array}$ & $\begin{array}{c}661 \\
31\end{array}$ & $\begin{array}{c}<0 . \\
1\end{array}$ & $\begin{array}{l}5 \\
7\end{array}$ & $\begin{array}{c}28 . \\
7\end{array}$ & $\begin{array}{c}12 \\
4\end{array}$ & 1.3 & 47 & $\begin{array}{c}6.0 \\
1\end{array}$ \\
\hline A236 & $\begin{array}{l}<0 . \\
1\end{array}$ & $\begin{array}{c}857 \\
35\end{array}$ & $\begin{array}{l}<0 . \\
1\end{array}$ & 528 & $\begin{array}{l}1 . \\
5\end{array}$ & $\begin{array}{c}0 . \\
1\end{array}$ & $\begin{array}{c}391 \\
48\end{array}$ & $\begin{array}{c}<0 . \\
1\end{array}$ & $\begin{array}{l}4 \\
8\end{array}$ & $\begin{array}{c}15 . \\
8\end{array}$ & 34 & 2.9 & 9 & $\begin{array}{c}4.4 \\
1\end{array}$ \\
\hline A237 & $\begin{array}{l}<0 . \\
1\end{array}$ & $\begin{array}{c}944 \\
22\end{array}$ & 0.2 & 63 & $\begin{array}{c}0 . \\
5\end{array}$ & $\begin{array}{c}0 . \\
1\end{array}$ & $\begin{array}{c}994 \\
89\end{array}$ & $\begin{array}{c}<0 . \\
1\end{array}$ & $\begin{array}{l}1 \\
7\end{array}$ & $\begin{array}{c}38 . \\
7\end{array}$ & 37 & $\begin{array}{c}<0 \\
5\end{array}$ & 48 & 2.7 \\
\hline A246 & $\begin{array}{l}<0 . \\
1\end{array}$ & $\begin{array}{c}>10 \\
\%\end{array}$ & 0.1 & 72 & $\begin{array}{c}0 . \\
5\end{array}$ & $\begin{array}{c}0 . \\
1\end{array}$ & $\begin{array}{c}941 \\
38\end{array}$ & $\begin{array}{c}<0 . \\
1\end{array}$ & $\begin{array}{l}1 \\
4\end{array}$ & $\begin{array}{c}26 . \\
1\end{array}$ & $\begin{array}{c}12 \\
7\end{array}$ & $\begin{array}{c}<0 . \\
5\end{array}$ & 37 & 2 \\
\hline
\end{tabular}

Table 2 (cont.) 


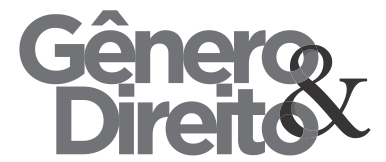

Periódico do Núcleo de Estudos e Pesquisas sobre Gênero e Direito Centro de Ciências Jurídicas - Universidade Federal da Paraíba V. 8 - $\mathrm{N}^{\circ} 03$ - Ano 2019 ISSN | 2179-7137 | http://periodicos.ufpb.br/ojs2/index.php/ged/index

\begin{tabular}{|c|c|c|c|c|c|c|c|c|c|c|c|c|c|c|}
\hline $\begin{array}{l}\text { Sam } \\
\text { ple }\end{array}$ & $\mathrm{Er}$ & $\mathrm{Eu}$ & $\mathrm{Fe}$ & $\mathrm{Gd}$ & Hf & In & $\mathrm{K}$ & $\mathrm{L}$ & $\begin{array}{l}\mathrm{L} \\
\mathrm{i}\end{array}$ & $\mathrm{Lu}$ & $\mathrm{Mg}$ & $\mathrm{Mn}$ & $\begin{array}{c}\mathrm{M} \\
\mathrm{o}\end{array}$ & $\mathrm{Na}$ \\
\hline $\mathrm{DL}^{*}$ & $\begin{array}{c}0.0 \\
5\end{array}$ & 0.1 & 100 & $\begin{array}{c}0.0 \\
5\end{array}$ & 0.5 & 0.5 & 100 & 1 & 1 & 0.1 & 100 & 5 & 0.1 & 100 \\
\hline A39 & $\begin{array}{c}0.8 \\
6\end{array}$ & $\begin{array}{c}0.7 \\
1\end{array}$ & $\begin{array}{c}158 \\
26\end{array}$ & $\begin{array}{c}1.7 \\
7\end{array}$ & $\begin{array}{c}<0 . \\
5\end{array}$ & $\begin{array}{l}<0 . \\
5\end{array}$ & $\begin{array}{c}322 \\
04\end{array}$ & 0 & $\begin{array}{l}2 \\
5\end{array}$ & $\begin{array}{c}0.1 \\
2\end{array}$ & $\begin{array}{c}562 \\
4\end{array}$ & $\begin{array}{c}38 \\
3\end{array}$ & 2.9 & $\begin{array}{c}240 \\
26\end{array}$ \\
\hline A61 & $\begin{array}{c}1.0 \\
7\end{array}$ & $\begin{array}{c}0.8 \\
1\end{array}$ & $\begin{array}{c}262 \\
08\end{array}$ & $\begin{array}{c}2.0 \\
7\end{array}$ & $\begin{array}{c}<0 . \\
5\end{array}$ & $\begin{array}{l}<0 . \\
5\end{array}$ & $\begin{array}{c}220 \\
74\end{array}$ & 0 & $\begin{array}{l}2 \\
9\end{array}$ & $\begin{array}{c}0.1 \\
7\end{array}$ & $\begin{array}{c}118 \\
59\end{array}$ & $\begin{array}{c}50 \\
2\end{array}$ & 2.1 & $\begin{array}{c}344 \\
84\end{array}$ \\
\hline A81 & $\begin{array}{c}2.2 \\
3\end{array}$ & $\begin{array}{c}1.0 \\
7\end{array}$ & $\begin{array}{c}373 \\
96\end{array}$ & $\begin{array}{c}3.5 \\
3\end{array}$ & $\begin{array}{c}0.6 \\
9\end{array}$ & $\begin{array}{l}<0 . \\
5\end{array}$ & $\begin{array}{c}194 \\
19\end{array}$ & $\begin{array}{l}3 \\
2\end{array}$ & $\begin{array}{l}5 \\
2\end{array}$ & $\begin{array}{c}0.3 \\
4\end{array}$ & $\begin{array}{l}>2 \\
\%\end{array}$ & $\begin{array}{l}10 \\
53\end{array}$ & 1.8 & $\begin{array}{c}338 \\
96\end{array}$ \\
\hline A105 & $\begin{array}{c}0.8 \\
8\end{array}$ & $\begin{array}{c}0.7 \\
3\end{array}$ & $\begin{array}{c}216 \\
02\end{array}$ & $\begin{array}{c}1.6 \\
4\end{array}$ & $\begin{array}{l}<0 . \\
5\end{array}$ & $\begin{array}{l}<0 . \\
5\end{array}$ & $\begin{array}{c}276 \\
95\end{array}$ & 5 & $\begin{array}{l}3 \\
2\end{array}$ & $\begin{array}{c}0.1 \\
5\end{array}$ & $\begin{array}{c}977 \\
8\end{array}$ & $\begin{array}{c}47 \\
0\end{array}$ & $\begin{array}{l}<0 . \\
1\end{array}$ & $\begin{array}{c}328 \\
66\end{array}$ \\
\hline A115 & $\begin{array}{c}1.4 \\
3\end{array}$ & $\begin{array}{c}2.1 \\
9\end{array}$ & $\begin{array}{r}307 \\
14\end{array}$ & $\begin{array}{c}2.9 \\
6\end{array}$ & $\begin{array}{l}<0 . \\
5\end{array}$ & $\begin{array}{l}<0 . \\
5\end{array}$ & $\begin{array}{c}596 \\
67\end{array}$ & 0 & 6 & $\begin{array}{c}0.2 \\
1\end{array}$ & $\begin{array}{c}149 \\
3\end{array}$ & $\begin{array}{c}57 \\
9\end{array}$ & $\begin{array}{l}<0 . \\
1\end{array}$ & $\begin{array}{c}311 \\
50\end{array}$ \\
\hline A137 & $\begin{array}{c}0.8 \\
9\end{array}$ & $\begin{array}{c}0.6 \\
1\end{array}$ & $\begin{array}{c}743 \\
77\end{array}$ & $\begin{array}{c}1.4 \\
8\end{array}$ & $\begin{array}{l}<0 . \\
5\end{array}$ & $\begin{array}{l}<0 . \\
5\end{array}$ & 652 & 4 & 4 & $\begin{array}{c}0.1 \\
1\end{array}$ & $\begin{array}{l}>2 \\
\%\end{array}$ & $\begin{array}{c}96 \\
5\end{array}$ & $\begin{array}{l}<0 . \\
1\end{array}$ & $\begin{array}{c}127 \\
13\end{array}$ \\
\hline A141 & $\begin{array}{c}1.4 \\
1\end{array}$ & $\begin{array}{c}1.6 \\
2\end{array}$ & $\begin{array}{c}170 \\
37\end{array}$ & $\begin{array}{c}2.5 \\
1\end{array}$ & $\begin{array}{l}<0 . \\
5\end{array}$ & $\begin{array}{l}<0 . \\
5\end{array}$ & $\begin{array}{c}420 \\
50\end{array}$ & 5 & $\begin{array}{l}1 \\
1\end{array}$ & 0.2 & $\begin{array}{c}233 \\
0\end{array}$ & $\begin{array}{c}31 \\
7\end{array}$ & $\begin{array}{l}<0 . \\
1\end{array}$ & $\begin{array}{c}340 \\
84\end{array}$ \\
\hline A162 & $\begin{array}{c}1.9 \\
1\end{array}$ & $\begin{array}{c}2.5 \\
3\end{array}$ & $\begin{array}{c}295 \\
54\end{array}$ & $\begin{array}{c}4.1 \\
2\end{array}$ & $\begin{array}{l}<0 . \\
5\end{array}$ & $\begin{array}{l}<0 . \\
5\end{array}$ & $\begin{array}{c}548 \\
41\end{array}$ & 9 & 7 & $\begin{array}{c}0.2 \\
2\end{array}$ & $\begin{array}{c}390 \\
8\end{array}$ & $\begin{array}{c}37 \\
3\end{array}$ & 1.1 & $\begin{array}{c}329 \\
01\end{array}$ \\
\hline A163 & $\begin{array}{c}2.3 \\
2\end{array}$ & $\begin{array}{c}1.2 \\
8\end{array}$ & $\begin{array}{c}424 \\
04\end{array}$ & 3.9 & 0.8 & $\begin{array}{l}<0 . \\
5\end{array}$ & $\begin{array}{c}188 \\
42\end{array}$ & 1 & $\begin{array}{l}2 \\
7\end{array}$ & $\begin{array}{c}0.3 \\
1\end{array}$ & $\begin{array}{l}>2 \\
\%\end{array}$ & $\begin{array}{c}86 \\
8\end{array}$ & 0.4 & $\begin{array}{c}313 \\
03\end{array}$ \\
\hline A174 & $\begin{array}{c}1.0 \\
8\end{array}$ & $\begin{array}{c}0.7 \\
1\end{array}$ & $\begin{array}{c}369 \\
61\end{array}$ & $\begin{array}{c}1.9 \\
8\end{array}$ & $\begin{array}{c}0.9 \\
8\end{array}$ & $\begin{array}{l}<0 . \\
5\end{array}$ & $\begin{array}{c}191 \\
17\end{array}$ & 7 & 7 & $\begin{array}{c}0.1 \\
9\end{array}$ & $\begin{array}{l}>2 \\
\%\end{array}$ & $\begin{array}{c}95 \\
3\end{array}$ & 1.1 & $\begin{array}{c}243 \\
71\end{array}$ \\
\hline A177 & $\begin{array}{c}0.7 \\
7\end{array}$ & $\begin{array}{c}0.5 \\
7\end{array}$ & $\begin{array}{c}262 \\
83\end{array}$ & $\begin{array}{c}1.1 \\
9\end{array}$ & $\begin{array}{c}0.8 \\
5\end{array}$ & $\begin{array}{l}<0 . \\
5\end{array}$ & 821 & 5 & 4 & $\begin{array}{c}0.1 \\
1\end{array}$ & $\begin{array}{l}>2 \\
\%\end{array}$ & $\begin{array}{c}46 \\
5\end{array}$ & 0.1 & $\begin{array}{c}118 \\
49\end{array}$ \\
\hline A193 & $\begin{array}{c}1.6 \\
5\end{array}$ & $\begin{array}{c}1.1 \\
4\end{array}$ & $\begin{array}{c}482 \\
62\end{array}$ & $\begin{array}{c}2.6 \\
7\end{array}$ & $\begin{array}{c}1.9 \\
4\end{array}$ & $\begin{array}{l}<0 . \\
5\end{array}$ & $\begin{array}{c}190 \\
5\end{array}$ & 9 & 8 & $\begin{array}{c}0.2 \\
4\end{array}$ & $\begin{array}{l}>2 \\
\%\end{array}$ & $\begin{array}{c}90 \\
6\end{array}$ & $\begin{array}{l}<0 . \\
1\end{array}$ & $\begin{array}{c}175 \\
51\end{array}$ \\
\hline A203 & $\begin{array}{c}1.7 \\
7\end{array}$ & $\begin{array}{c}1.0 \\
3\end{array}$ & $\begin{array}{c}508 \\
61\end{array}$ & $\begin{array}{c}2.9 \\
4\end{array}$ & 2.2 & $\begin{array}{l}<0 . \\
5\end{array}$ & $\begin{array}{c}156 \\
7\end{array}$ & 0 & $\begin{array}{l}1 \\
7\end{array}$ & $\begin{array}{c}0.2 \\
6\end{array}$ & $\begin{array}{l}>2 \\
\%\end{array}$ & $\begin{array}{c}95 \\
5\end{array}$ & $\begin{array}{l}<0 . \\
1\end{array}$ & $\begin{array}{c}100 \\
77\end{array}$ \\
\hline A225 & $\begin{array}{c}4.4 \\
6\end{array}$ & $\begin{array}{c}1.8 \\
7\end{array}$ & $\begin{array}{c}677 \\
83\end{array}$ & $\begin{array}{c}6.4 \\
8\end{array}$ & $\begin{array}{c}1.2 \\
2\end{array}$ & $\begin{array}{l}<0 . \\
5\end{array}$ & $\begin{array}{c}105 \\
61\end{array}$ & 7 & $\begin{array}{l}1 \\
9\end{array}$ & $\begin{array}{c}0.6 \\
2\end{array}$ & $\begin{array}{l}>2 \\
\%\end{array}$ & $\begin{array}{l}15 \\
89\end{array}$ & 0.1 & $\begin{array}{c}300 \\
67\end{array}$ \\
\hline A230 & $\begin{array}{c}3.3 \\
8\end{array}$ & $\begin{array}{c}1.4 \\
2\end{array}$ & $\begin{array}{c}590 \\
29\end{array}$ & $\begin{array}{c}5.1 \\
8\end{array}$ & $\begin{array}{c}0.8 \\
2\end{array}$ & $\begin{array}{l}<0 . \\
5\end{array}$ & $\begin{array}{c}164 \\
98\end{array}$ & $\begin{array}{l}2 \\
7\end{array}$ & $\begin{array}{l}1 \\
9\end{array}$ & $\begin{array}{c}0.4 \\
6\end{array}$ & $\begin{array}{l}>2 \\
\%\end{array}$ & $\begin{array}{l}13 \\
86\end{array}$ & $\begin{array}{l}<0 . \\
1\end{array}$ & $\begin{array}{c}281 \\
26\end{array}$ \\
\hline $\begin{array}{c}\text { A232 } \\
\text { A }\end{array}$ & $\begin{array}{c}3.4 \\
6\end{array}$ & $\begin{array}{c}1.5 \\
1\end{array}$ & $\begin{array}{c}594 \\
67\end{array}$ & $\begin{array}{c}5.2 \\
5\end{array}$ & $\begin{array}{c}1.2 \\
8\end{array}$ & $\begin{array}{l}<0 . \\
5\end{array}$ & $\begin{array}{c}110 \\
83\end{array}$ & 8 & $\begin{array}{l}2 \\
4\end{array}$ & $\begin{array}{c}0.4 \\
8\end{array}$ & $\begin{array}{l}>2 \\
\%\end{array}$ & $\begin{array}{l}14 \\
48\end{array}$ & & $\begin{array}{c}298 \\
83\end{array}$ \\
\hline
\end{tabular}




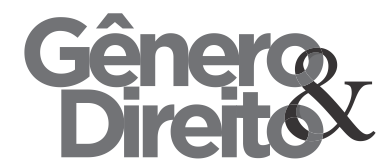

Periódico do Núcleo de Estudos e Pesquisas sobre Gênero e Direito

Centro de Ciências Jurídicas - Universidade Federal da Paraíba V. 8 - $\mathrm{N}^{\circ} 03$ - Ano 2019

ISSN | 2179-7137 | http://periodicos.ufpb.br/ojs2/index.php/ged/index

\begin{tabular}{|c|c|c|c|c|c|c|c|c|c|c|c|c|c|c|}
\hline $\begin{array}{c}\text { A232 } \\
\text { B }\end{array}$ & $\begin{array}{c}3.3 \\
2\end{array}$ & $\begin{array}{c}1.5 \\
7\end{array}$ & $\begin{array}{c}595 \\
30\end{array}$ & $\begin{array}{c}5.0 \\
8\end{array}$ & $\begin{array}{c}1.2 \\
8\end{array}$ & $\begin{array}{l}<0 . \\
5\end{array}$ & $\begin{array}{c}109 \\
58\end{array}$ & $\begin{array}{c}2 \\
6\end{array}$ & $\begin{array}{c}2 \\
4\end{array}$ & $\begin{array}{c}0.4 \\
8\end{array}$ & $\begin{array}{l}> \\
\%\end{array}$ & $\begin{array}{c}14 \\
37\end{array}$ & $\begin{array}{c}<0 . \\
1\end{array}$ & $\begin{array}{c}302 \\
85\end{array}$ \\
\hline A236 & 2.3 & 1.1 & 422 & 3.6 & $<0$. & $<0$. & 187 & 2 & 2 & 0.3 & 154 & 89 & 0.3 & 291 \\
& 9 & 4 & 86 & 1 & 5 & 5 & 83 & 6 & 1 & 1 & 70 & 3 & & 82 \\
\hline A237 & 1.2 & 0.8 & 517 & 1.8 & 0.6 & $<0$. & 109 & 6 & 5 & 0.1 & $>2$ & 10 & $<0$. & 215 \\
& & & 85 & 9 & 8 & 5 & 0 & & & 6 & $\%$ & 28 & 1 & 23 \\
\hline A246 & 0.8 & 0.8 & 370 & 1.2 & $<0$. & $<0$. & 128 & 4 & 5 & 0.1 & $>2$ & 81 & $<0$. & 256 \\
& 2 & 6 & 99 & 6 & 5 & 5 & 7 & & & 2 & $\%$ & 5 & 1 & 76 \\
\hline
\end{tabular}

$<\mathrm{DL}^{*}=$ below detection limit

Table 2 (cont.)

\begin{tabular}{|c|c|c|c|c|c|c|c|c|c|c|c|c|c|c|}
\hline $\begin{array}{c}\text { Samp } \\
\text { le }\end{array}$ & $\mathrm{Nb}$ & $\mathrm{Nd}$ & $\mathrm{Ni}$ & $\mathrm{P}$ & $\begin{array}{l}\mathrm{P} \\
\mathrm{b}\end{array}$ & $\operatorname{Pr}$ & $\mathrm{Rb}$ & $\mathrm{S}$ & $\mathrm{Sb}$ & $\mathrm{Sc}$ & $\mathrm{Se}$ & $\mathrm{Sm}$ & $\begin{array}{l}\mathrm{S} \\
\mathrm{n}\end{array}$ & $\mathrm{Sr}$ \\
\hline $\mathrm{DL}^{*}$ & 1 & 0.5 & 1 & 10 & 1 & $\begin{array}{c}0.0 \\
5\end{array}$ & 1 & 50 & 0.5 & 0.5 & 0.5 & $\begin{array}{c}0.0 \\
2\end{array}$ & $\begin{array}{l}0 . \\
1\end{array}$ & 1 \\
\hline A39 & $\begin{array}{c}54 . \\
9\end{array}$ & $\begin{array}{c}13 . \\
7\end{array}$ & 7 & 504 & 9 & $\begin{array}{c}4.1 \\
8\end{array}$ & $\begin{array}{c}13 \\
4\end{array}$ & $<50$ & 2.1 & 4.1 & $\begin{array}{c}2.0 \\
3\end{array}$ & $\begin{array}{c}2.2 \\
3\end{array}$ & $\begin{array}{l}2 . \\
9\end{array}$ & $\begin{array}{c}28 \\
5\end{array}$ \\
\hline A61 & $\begin{array}{c}57 . \\
8\end{array}$ & $\begin{array}{c}14 . \\
4\end{array}$ & 10 & 779 & 7 & $\begin{array}{c}4.2 \\
8\end{array}$ & 88 & 73 & 1.7 & 7.7 & $\begin{array}{c}2.1 \\
2\end{array}$ & $\begin{array}{c}2.5 \\
5\end{array}$ & $\begin{array}{l}2 . \\
4\end{array}$ & $\begin{array}{c}41 \\
0\end{array}$ \\
\hline A81 & $\begin{array}{c}12 \\
1\end{array}$ & $\begin{array}{c}21 . \\
9\end{array}$ & 24 & $\begin{array}{c}134 \\
6\end{array}$ & 8 & $\begin{array}{c}6.2 \\
6\end{array}$ & 86 & 89 & $\begin{array}{l}<0 . \\
5\end{array}$ & $\begin{array}{c}19 . \\
4\end{array}$ & $\begin{array}{c}1.8 \\
2\end{array}$ & $\begin{array}{c}3.9 \\
3\end{array}$ & $\begin{array}{l}2 . \\
7\end{array}$ & $\begin{array}{c}34 \\
3\end{array}$ \\
\hline A105 & $\begin{array}{c}74 . \\
7\end{array}$ & $\begin{array}{c}11 . \\
9\end{array}$ & 11 & 727 & 9 & $\begin{array}{c}3.5 \\
2\end{array}$ & 87 & 58 & $\begin{array}{l}<0 . \\
5\end{array}$ & 5.4 & $\begin{array}{c}1.7 \\
7\end{array}$ & $\begin{array}{c}2.1 \\
1\end{array}$ & 1 & $\begin{array}{c}42 \\
2\end{array}$ \\
\hline A115 & $\begin{array}{c}31 . \\
5\end{array}$ & $\begin{array}{c}23 . \\
2\end{array}$ & 3 & 534 & $\begin{array}{l}1 \\
2\end{array}$ & $\begin{array}{c}6.7 \\
7\end{array}$ & 79 & 125 & $\begin{array}{l}<0 . \\
5\end{array}$ & 8.4 & $\begin{array}{c}1.5 \\
4\end{array}$ & $\begin{array}{c}3.8 \\
9\end{array}$ & 1 & $\begin{array}{c}19 \\
9\end{array}$ \\
\hline A137 & $\begin{array}{c}12 . \\
6\end{array}$ & 5.2 & 40 & 130 & 9 & $\begin{array}{c}1.0 \\
4\end{array}$ & 5 & 154 & $\begin{array}{l}<0 . \\
5\end{array}$ & $\begin{array}{c}58 . \\
5\end{array}$ & $\begin{array}{c}0.7 \\
4\end{array}$ & $\begin{array}{c}1.3 \\
2\end{array}$ & $\begin{array}{l}0 . \\
2\end{array}$ & $\begin{array}{c}56 \\
7\end{array}$ \\
\hline A141 & $\begin{array}{c}44 . \\
9\end{array}$ & 15 & 5 & 479 & $\begin{array}{l}1 \\
5\end{array}$ & $\begin{array}{c}4.1 \\
1\end{array}$ & 76 & 55 & 0.6 & 6.5 & $\begin{array}{c}1.3 \\
9\end{array}$ & $\begin{array}{c}3.1 \\
5\end{array}$ & $\begin{array}{l}1 . \\
2\end{array}$ & $\begin{array}{c}26 \\
2\end{array}$ \\
\hline A162 & $\begin{array}{c}62 . \\
2\end{array}$ & $\begin{array}{c}20 . \\
7\end{array}$ & 3 & 714 & $\begin{array}{l}1 \\
9\end{array}$ & $\begin{array}{c}5.1 \\
1\end{array}$ & 95 & 53 & 0.5 & 9.5 & $\begin{array}{c}1.4 \\
2\end{array}$ & $\begin{array}{c}4.6 \\
5\end{array}$ & $\begin{array}{l}1 . \\
5\end{array}$ & $\begin{array}{c}36 \\
0\end{array}$ \\
\hline A163 & $\begin{array}{c}51 . \\
6\end{array}$ & $\begin{array}{c}21 . \\
4\end{array}$ & 37 & $\begin{array}{c}121 \\
7\end{array}$ & $\begin{array}{l}1 \\
0\end{array}$ & $\begin{array}{c}5.4 \\
7\end{array}$ & 58 & 93 & 0.8 & $\begin{array}{c}21 . \\
7\end{array}$ & $\begin{array}{c}1.5 \\
6\end{array}$ & $\begin{array}{c}4.4 \\
2\end{array}$ & $\begin{array}{l}1 . \\
4\end{array}$ & $\begin{array}{c}64 \\
1\end{array}$ \\
\hline A174 & $\begin{array}{c}36 . \\
3\end{array}$ & $\begin{array}{c}12 . \\
6\end{array}$ & 99 & 772 & 4 & $\begin{array}{c}3.4 \\
7\end{array}$ & 41 & 52 & $\begin{array}{l}<0 . \\
5\end{array}$ & $\begin{array}{c}21 . \\
2\end{array}$ & $\begin{array}{c}1.0 \\
7\end{array}$ & $\begin{array}{c}2.2 \\
6\end{array}$ & 1. & $\begin{array}{c}56 \\
7\end{array}$ \\
\hline
\end{tabular}


Periódico do Núcleo de Estudos e Pesquisas sobre Gênero e Direito Centro de Ciências Jurídicas - Universidade Federal da Paraíba V. 8 - $\mathrm{N}^{\circ} 03$ - Ano 2019 ISSN | 2179-7137 | http://periodicos.ufpb.br/ojs2/index.php/ged/index

\begin{tabular}{|c|c|c|c|c|c|c|c|c|c|c|c|c|c|c|}
\hline A177 & $\begin{array}{c}11 . \\
4\end{array}$ & 5.2 & $\begin{array}{c}13 \\
0\end{array}$ & 159 & $\begin{array}{l}< \\
1\end{array}$ & 1.1 & 6 & 669 & $\begin{array}{l}<0 . \\
5\end{array}$ & $\begin{array}{c}34 . \\
7\end{array}$ & $\begin{array}{c}0.6 \\
3\end{array}$ & $\begin{array}{c}1.1 \\
9\end{array}$ & $\begin{array}{c}0 . \\
4\end{array}$ & $\begin{array}{c}78 \\
0\end{array}$ \\
\hline A193 & 5.1 & $\begin{array}{c}10 . \\
3\end{array}$ & 29 & 449 & 9 & $\begin{array}{c}2.1 \\
4\end{array}$ & 8 & 58 & $\begin{array}{l}<0 . \\
5\end{array}$ & $\begin{array}{c}37 . \\
6\end{array}$ & $\begin{array}{c}1.3 \\
1\end{array}$ & $\begin{array}{c}2.5 \\
8\end{array}$ & $\begin{array}{l}0 . \\
3\end{array}$ & $\begin{array}{c}64 \\
2\end{array}$ \\
\hline A203 & $<1$ & $\begin{array}{c}11 . \\
6\end{array}$ & 71 & 921 & 3 & $\begin{array}{c}2.4 \\
3\end{array}$ & 8 & 156 & $\begin{array}{l}<0 . \\
5\end{array}$ & $\begin{array}{c}43 . \\
4\end{array}$ & $\begin{array}{c}1.3 \\
6\end{array}$ & 2.8 & $\begin{array}{l}1 . \\
7\end{array}$ & $\begin{array}{c}48 \\
9\end{array}$ \\
\hline A225 & $\begin{array}{c}28 \\
4\end{array}$ & $\begin{array}{c}29 . \\
5\end{array}$ & 9 & $\begin{array}{c}128 \\
9\end{array}$ & 9 & 6.9 & 34 & 128 & $\begin{array}{l}<0 . \\
5\end{array}$ & $\begin{array}{c}39 . \\
6\end{array}$ & $\begin{array}{c}2.3 \\
2\end{array}$ & $\begin{array}{c}6.7 \\
9\end{array}$ & $\begin{array}{l}1 . \\
7\end{array}$ & $\begin{array}{c}50 \\
8\end{array}$ \\
\hline A230 & $\begin{array}{c}20 . \\
8\end{array}$ & 23 & 12 & $\begin{array}{c}119 \\
6\end{array}$ & $\begin{array}{l}1 \\
3\end{array}$ & $\begin{array}{c}5.4 \\
5\end{array}$ & 62 & 140 & $\begin{array}{l}<0 . \\
5\end{array}$ & $\begin{array}{c}28 . \\
1\end{array}$ & $\begin{array}{c}1.7 \\
5\end{array}$ & 5.2 & $\begin{array}{l}1 . \\
5\end{array}$ & $\begin{array}{c}41 \\
1\end{array}$ \\
\hline $\begin{array}{c}\mathrm{A} 232 \\
\text { A }\end{array}$ & $\begin{array}{c}21 . \\
4\end{array}$ & $\begin{array}{c}25 . \\
3\end{array}$ & 44 & $\begin{array}{c}123 \\
6\end{array}$ & 9 & $\begin{array}{c}6.1 \\
2\end{array}$ & 40 & 116 & 2.4 & $\begin{array}{c}35 . \\
6\end{array}$ & $\begin{array}{c}1.5 \\
1\end{array}$ & $\begin{array}{c}5.5 \\
2\end{array}$ & $\begin{array}{l}2 . \\
3\end{array}$ & $\begin{array}{c}48 \\
7\end{array}$ \\
\hline $\begin{array}{c}\mathrm{A} 232 \\
\text { B }\end{array}$ & $\begin{array}{c}22 . \\
6\end{array}$ & $\begin{array}{c}24 . \\
9\end{array}$ & 42 & $\begin{array}{c}120 \\
9\end{array}$ & 9 & $\begin{array}{c}6.0 \\
1\end{array}$ & 40 & 100 & $\begin{array}{c}<0 . \\
5\end{array}$ & $\begin{array}{c}35 . \\
7\end{array}$ & $\begin{array}{c}3.4 \\
6\end{array}$ & $\begin{array}{c}5.3 \\
5\end{array}$ & $\begin{array}{l}2 . \\
3\end{array}$ & $\begin{array}{c}48 \\
5\end{array}$ \\
\hline A236 & $\begin{array}{c}20 . \\
8\end{array}$ & $\begin{array}{c}18 . \\
7\end{array}$ & 9 & $\begin{array}{c}104 \\
1\end{array}$ & 7 & $\begin{array}{c}4.6 \\
9\end{array}$ & 72 & 107 & $\begin{array}{l}<0 . \\
5\end{array}$ & 17 & $\begin{array}{c}2.2 \\
7\end{array}$ & 3.9 & $\begin{array}{l}1 . \\
2\end{array}$ & $\begin{array}{c}42 \\
2\end{array}$ \\
\hline A237 & 3.8 & 6.6 & 39 & 230 & 2 & $\begin{array}{c}1.4 \\
1\end{array}$ & 5 & $\begin{array}{c}152 \\
0\end{array}$ & $\begin{array}{l}<0 . \\
5\end{array}$ & $\begin{array}{c}53 . \\
1\end{array}$ & $\begin{array}{c}1.2 \\
1\end{array}$ & $\begin{array}{c}1.6 \\
6\end{array}$ & $\begin{array}{l}0 . \\
3\end{array}$ & $\begin{array}{c}52 \\
1\end{array}$ \\
\hline A246 & 5.3 & 5 & 47 & 121 & 6 & 1 & 6 & 986 & $\begin{array}{l}<0 . \\
5\end{array}$ & $\begin{array}{c}34 . \\
7\end{array}$ & $\begin{array}{c}1.4 \\
9\end{array}$ & 1.2 & $\begin{array}{l}0 . \\
3\end{array}$ & $\begin{array}{c}52 \\
1\end{array}$ \\
\hline
\end{tabular}

Table 2 (cont.)

\begin{tabular}{|c|c|c|c|c|c|c|c|c|c|c|c|c|c|c|}
\hline$\underset{\text { le }}{\text { Samp }}$ & $\mathrm{Ta}$ & $\mathrm{Tb}$ & $\mathrm{Te}$ & $\mathrm{Th}$ & $\mathrm{Ti}$ & $\mathrm{Tl}$ & $\mathrm{Tm}$ & $\mathrm{U}$ & $\mathrm{V}$ & W & $\mathrm{Y}$ & $\mathrm{Yb}$ & $\mathrm{Zn}$ & $\mathrm{Zr}$ \\
\hline $\mathrm{DL}^{*}$ & 0.1 & 0.1 & 0.1 & 0.1 & 10 & 0.1 & 0.1 & 0.1 & 1 & 1 & 0.5 & $\begin{array}{c}0.0 \\
5\end{array}$ & 1 & 5 \\
\hline A39 & $\begin{array}{c}1.6 \\
6\end{array}$ & $\begin{array}{c}0.3 \\
2\end{array}$ & $\begin{array}{c}0.4 \\
2\end{array}$ & $\begin{array}{c}13.9 \\
3\end{array}$ & $\begin{array}{c}210 \\
8\end{array}$ & $\begin{array}{c}0.6 \\
1\end{array}$ & $\begin{array}{c}0.1 \\
4\end{array}$ & 2.6 & 40 & $\begin{array}{l}< \\
1\end{array}$ & 8.7 & 0.5 & 27 & 15 \\
\hline A61 & 1 & $\begin{array}{c}0.3 \\
6\end{array}$ & $\begin{array}{c}0.1 \\
9\end{array}$ & 9.86 & $\begin{array}{c}401 \\
4\end{array}$ & $\begin{array}{c}0.4 \\
1\end{array}$ & $\begin{array}{c}0.1 \\
7\end{array}$ & 1.2 & 74 & $\begin{array}{l}< \\
1\end{array}$ & $\begin{array}{c}10 . \\
6\end{array}$ & 1.2 & 40 & 12 \\
\hline A81 & $\begin{array}{c}2.7 \\
1\end{array}$ & $\begin{array}{c}0.5 \\
7\end{array}$ & $\begin{array}{c}0.1 \\
2\end{array}$ & 7.03 & $\begin{array}{c}605 \\
9\end{array}$ & $\begin{array}{c}0.4 \\
1\end{array}$ & $\begin{array}{c}0.3 \\
3\end{array}$ & 2.1 & $\begin{array}{c}13 \\
2\end{array}$ & $\begin{array}{l}< \\
1\end{array}$ & 20 & 3.4 & 56 & 23 \\
\hline A105 & $\begin{array}{c}1.7 \\
5\end{array}$ & $\begin{array}{c}0.3 \\
1\end{array}$ & $\begin{array}{c}0.2 \\
4\end{array}$ & 8.44 & $\begin{array}{c}322 \\
7\end{array}$ & $\begin{array}{c}0.3 \\
4\end{array}$ & $\begin{array}{c}0.1 \\
5\end{array}$ & $\begin{array}{c}1.3 \\
3\end{array}$ & 63 & $\begin{array}{l}< \\
1\end{array}$ & 8.8 & 0.8 & 35 & 14 \\
\hline A115 & $\begin{array}{c}0.8 \\
1\end{array}$ & $\begin{array}{c}0.4 \\
7\end{array}$ & $\begin{array}{c}<0 . \\
1\end{array}$ & 5.83 & $\begin{array}{c}294 \\
9\end{array}$ & $\begin{array}{c}0.3 \\
4\end{array}$ & $\begin{array}{c}0.2 \\
1\end{array}$ & 0.4 & 19 & $\begin{array}{l}< \\
1\end{array}$ & 14 & 1.5 & 56 & 13 \\
\hline
\end{tabular}




\begin{tabular}{|c|c|c|c|c|c|c|c|c|c|c|c|c|c|c|}
\hline A137 & $\begin{array}{c}0.4 \\
7\end{array}$ & $\begin{array}{c}0.3 \\
2\end{array}$ & $\begin{array}{c}0.5 \\
5\end{array}$ & 0.36 & $\begin{array}{c}954 \\
1\end{array}$ & $\begin{array}{c}<0 . \\
1\end{array}$ & $\begin{array}{c}0.1 \\
4\end{array}$ & $\begin{array}{c}<0 . \\
1\end{array}$ & $\begin{array}{c}47 \\
9\end{array}$ & $\begin{array}{l}< \\
1\end{array}$ & 9.2 & 3 & 57 & 33 \\
\hline A141 & $\begin{array}{c}1.1 \\
4\end{array}$ & $\begin{array}{c}0.4 \\
2\end{array}$ & $\begin{array}{l}<0 . \\
1\end{array}$ & 6.91 & $\begin{array}{c}255 \\
5\end{array}$ & $\begin{array}{c}0.3 \\
9\end{array}$ & 0.2 & 0.8 & 21 & 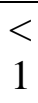 & $\begin{array}{c}13 . \\
7\end{array}$ & 1.3 & 25 & 12 \\
\hline A162 & $\begin{array}{c}2.1 \\
1\end{array}$ & $\begin{array}{c}0.6 \\
5\end{array}$ & $\begin{array}{l}<0 . \\
1\end{array}$ & 8.53 & $\begin{array}{c}439 \\
8\end{array}$ & $\begin{array}{c}0.3 \\
8\end{array}$ & $\begin{array}{c}0.2 \\
4\end{array}$ & 2.5 & 26 & 1 & $\begin{array}{c}18 . \\
6\end{array}$ & 2 & 31 & 24 \\
\hline A163 & $\begin{array}{c}1.4 \\
2\end{array}$ & $\begin{array}{c}0.6 \\
3\end{array}$ & $\begin{array}{c}0.1 \\
5\end{array}$ & 6.8 & $\begin{array}{c}669 \\
8\end{array}$ & $\begin{array}{c}0.2 \\
6\end{array}$ & 0.3 & 2.1 & $\begin{array}{c}15 \\
5\end{array}$ & 1 & $\begin{array}{c}21 . \\
7\end{array}$ & 3.4 & 70 & 31 \\
\hline A174 & $\begin{array}{c}0.9 \\
4\end{array}$ & $\begin{array}{c}0.3 \\
4\end{array}$ & $\begin{array}{c}0.2 \\
5\end{array}$ & 9.84 & $\begin{array}{c}493 \\
8\end{array}$ & $\begin{array}{c}0.1 \\
5\end{array}$ & $\begin{array}{c}0.1 \\
7\end{array}$ & 1.7 & $\begin{array}{c}11 \\
4\end{array}$ & 1 & $\begin{array}{c}10 . \\
8\end{array}$ & 1.7 & 68 & 28 \\
\hline A177 & $\begin{array}{c}0.4 \\
8\end{array}$ & $\begin{array}{c}0.2 \\
8\end{array}$ & $\begin{array}{c}0.6 \\
4\end{array}$ & 0.54 & $\begin{array}{c}384 \\
8\end{array}$ & $\begin{array}{l}<0 . \\
1\end{array}$ & $\begin{array}{c}0.1 \\
2\end{array}$ & $\begin{array}{c}0.2 \\
7\end{array}$ & $\begin{array}{c}12 \\
5\end{array}$ & 1 & 8 & 1 & 24 & 54 \\
\hline A193 & $\begin{array}{c}0.2 \\
9\end{array}$ & $\begin{array}{c}0.4 \\
9\end{array}$ & $\begin{array}{c}0.6 \\
3\end{array}$ & 0.8 & $\begin{array}{c}616 \\
4\end{array}$ & $\begin{array}{l}<0 . \\
1\end{array}$ & $\begin{array}{c}0.2 \\
2\end{array}$ & 0.2 & $\begin{array}{c}12 \\
1\end{array}$ & 1 & $\begin{array}{c}15 . \\
7\end{array}$ & 2.4 & 54 & $\begin{array}{c}10 \\
0\end{array}$ \\
\hline A203 & $\begin{array}{c}0.2 \\
1\end{array}$ & $\begin{array}{c}0.5 \\
3\end{array}$ & $\begin{array}{c}0.2 \\
8\end{array}$ & 1.04 & $\begin{array}{c}646 \\
9\end{array}$ & $\begin{array}{l}<0 . \\
1\end{array}$ & $\begin{array}{c}0.2 \\
6\end{array}$ & 0.4 & $\begin{array}{c}15 \\
5\end{array}$ & 1 & $\begin{array}{c}16 . \\
6\end{array}$ & 2.7 & 59 & $\begin{array}{c}10 \\
2\end{array}$ \\
\hline A225 & $\begin{array}{c}0.7 \\
6\end{array}$ & $\begin{array}{c}1.0 \\
4\end{array}$ & $\begin{array}{c}0.1 \\
8\end{array}$ & 1.86 & $\begin{array}{c}788 \\
9\end{array}$ & $\begin{array}{c}0.1 \\
4\end{array}$ & $\begin{array}{c}0.5 \\
6\end{array}$ & 0.4 & $\begin{array}{c}30 \\
9\end{array}$ & 1 & $\begin{array}{c}41 . \\
4\end{array}$ & 8.2 & $\begin{array}{c}10 \\
8\end{array}$ & 46 \\
\hline A230 & 0.6 & $\begin{array}{c}0.8 \\
2\end{array}$ & $\begin{array}{c}0.1 \\
8\end{array}$ & 5.64 & $\begin{array}{c}712 \\
0\end{array}$ & $\begin{array}{c}0.2 \\
5\end{array}$ & $\begin{array}{c}0.4 \\
7\end{array}$ & $\begin{array}{c}1.3 \\
1\end{array}$ & $\begin{array}{c}22 \\
1\end{array}$ & 1 & $\begin{array}{c}33 . \\
3\end{array}$ & 5.9 & 95 & 32 \\
\hline $\begin{array}{c}\mathrm{A} 232 \\
\text { A }\end{array}$ & $\begin{array}{c}0.5 \\
5\end{array}$ & $\begin{array}{c}0.8 \\
1\end{array}$ & $\begin{array}{c}0.1 \\
3\end{array}$ & 1.66 & $\begin{array}{c}625 \\
0\end{array}$ & $\begin{array}{c}0.1 \\
4\end{array}$ & $\begin{array}{c}0.4 \\
7\end{array}$ & 0.4 & $\begin{array}{c}23 \\
5\end{array}$ & 1 & $\begin{array}{c}34 . \\
1\end{array}$ & 6.4 & 97 & 46 \\
\hline $\begin{array}{c}\mathrm{A} 232 \\
\text { B }\end{array}$ & $\begin{array}{c}0.6 \\
1\end{array}$ & $\begin{array}{c}0.8 \\
4\end{array}$ & $\begin{array}{l}<0 . \\
1\end{array}$ & 1.38 & $\begin{array}{c}619 \\
6\end{array}$ & $\begin{array}{c}0.1 \\
5\end{array}$ & $\begin{array}{c}0.4 \\
6\end{array}$ & 0.3 & $\begin{array}{c}23 \\
2\end{array}$ & 1 & $\begin{array}{c}33 . \\
1\end{array}$ & 6.2 & $\begin{array}{c}11 \\
4\end{array}$ & 47 \\
\hline A236 & $\begin{array}{c}0.6 \\
3\end{array}$ & 0.6 & $\begin{array}{l}<0 . \\
1\end{array}$ & 6.51 & $\begin{array}{c}541 \\
7\end{array}$ & $\begin{array}{c}0.3 \\
2\end{array}$ & $\begin{array}{c}0.3 \\
3\end{array}$ & 1.5 & $\begin{array}{c}13 \\
4\end{array}$ & 1 & $\begin{array}{c}22 . \\
6\end{array}$ & 3.7 & 72 & 18 \\
\hline A237 & 0.3 & $\begin{array}{c}0.3 \\
6\end{array}$ & 0.3 & 0.3 & $\begin{array}{c}680 \\
2\end{array}$ & $\begin{array}{l}<0 . \\
1\end{array}$ & $\begin{array}{c}0.1 \\
8\end{array}$ & 0.1 & $\begin{array}{c}24 \\
7\end{array}$ & 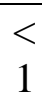 & $\begin{array}{c}12 . \\
5\end{array}$ & 2.4 & 51 & 48 \\
\hline A246 & $\begin{array}{c}0.3 \\
6\end{array}$ & $\begin{array}{c}0.2 \\
9\end{array}$ & $\begin{array}{c}<0 . \\
1\end{array}$ & 0.28 & $\begin{array}{c}394 \\
0\end{array}$ & $\begin{array}{l}<0 . \\
1\end{array}$ & $\begin{array}{c}0.1 \\
5\end{array}$ & 0.1 & $\begin{array}{c}11 \\
8\end{array}$ & 1 & 9.2 & 1.2 & 46 & 33 \\
\hline
\end{tabular}

Table 3. Microprobe analysis results for the plagioclase from the rocks in the study region.

\begin{tabular}{|c|c|c|c|c|c|c|c|c|c|c|c|c|}
\hline $\begin{array}{c}\text { Sam } \\
\text { ple }\end{array}$ & $\begin{array}{c}\text { A6- } \\
2\end{array}$ & $\begin{array}{c}\text { A6- } \\
\text { A6- }\end{array}$ & 5 & A6- & A23 & A23 & A23 & A23 & A23 & A23 & A15 & A15 \\
-1 & -2 & -3 & -4 & -7 & -8 & $4-1$ & $4-4$ \\
\hline
\end{tabular}




\begin{tabular}{|c|c|c|c|c|c|c|c|c|c|c|c|c|}
\hline $\mathrm{SiO}_{2}$ & $\begin{array}{c}58.4 \\
6\end{array}$ & $\begin{array}{c}61.8 \\
2\end{array}$ & $\begin{array}{c}57.0 \\
8\end{array}$ & $\begin{array}{c}70.4 \\
7\end{array}$ & 57.9 & $\begin{array}{c}64.0 \\
5\end{array}$ & $\begin{array}{c}59.7 \\
4\end{array}$ & $\begin{array}{c}62.3 \\
3\end{array}$ & $\begin{array}{c}56.2 \\
5\end{array}$ & $\begin{array}{c}62.0 \\
1\end{array}$ & $\begin{array}{c}58.7 \\
2\end{array}$ & 56.3 \\
\hline $\mathrm{Al}_{2}$ & 23.2 & 22.5 & 22.2 & 20.7 & 20.7 & 19.1 & 21.8 & 21.5 & 22.4 & 21.6 & 20.8 & 22.1 \\
\hline $\mathrm{O}_{3}$ & 3 & 8 & 6 & 6 & 7 & 1 & 7 & 6 & 4 & 3 & 4 & 6 \\
\hline $\begin{array}{c}\mathrm{Fe}_{2} \\
\mathrm{O}_{3}\end{array}$ & 0.43 & 0.44 & 0.41 & 0.28 & 0.4 & 0.31 & 0.23 & 0.22 & 0.44 & 0.25 & 0.17 & 0.31 \\
\hline $\mathrm{TiO}_{2}$ & - & - & - & - & - & - & - & - & - & - & - & - \\
\hline $\begin{array}{c}\mathrm{Na}_{2} \\
\mathrm{O}\end{array}$ & 4.3 & - & 7.27 & 3.03 & 8.58 & 6.03 & 6.28 & 5.43 & 5.75 & 4.96 & 6.82 & 7.89 \\
\hline $\mathrm{CaO}$ & $\begin{array}{c}12.9 \\
8\end{array}$ & $\begin{array}{c}14.1 \\
2\end{array}$ & $\begin{array}{c}11.7 \\
5\end{array}$ & 4.88 & $\begin{array}{c}11.7 \\
9\end{array}$ & 9.92 & $\begin{array}{c}11.2 \\
2\end{array}$ & $\begin{array}{c}10.0 \\
5\end{array}$ & $\begin{array}{c}14.9 \\
2\end{array}$ & $\begin{array}{c}10.9 \\
2\end{array}$ & 12 & $\begin{array}{c}11.4 \\
8\end{array}$ \\
\hline $\mathrm{K}_{2} \mathrm{O}$ & 0.47 & 0.9 & 0.66 & 0.61 & 0.39 & 0.36 & 0.33 & 0.28 & 0.2 & 0.23 & 0.63 & 0.55 \\
\hline $\begin{array}{c}\mathrm{Mn} \\
\mathrm{O}\end{array}$ & - & - & - & - & - & - & - & - & - & - & - & - \\
\hline $\begin{array}{c}\text { Tota } \\
1\end{array}$ & $\begin{array}{c}99.8 \\
7\end{array}$ & $\begin{array}{c}99.8 \\
6\end{array}$ & $\begin{array}{c}99.4 \\
3\end{array}$ & $\begin{array}{c}100 . \\
01\end{array}$ & $\begin{array}{c}99.8 \\
3\end{array}$ & $\begin{array}{c}99.7 \\
8\end{array}$ & $\begin{array}{c}99.6 \\
7\end{array}$ & $\begin{array}{c}99.8 \\
7\end{array}$ & 100 & 100 & $\begin{array}{c}99.1 \\
8\end{array}$ & $\begin{array}{c}98.6 \\
9\end{array}$ \\
\hline $\mathrm{Si}$ & $\begin{array}{c}10.5 \\
78\end{array}$ & $\begin{array}{c}11.0 \\
04\end{array}$ & $\begin{array}{c}10.5 \\
07\end{array}$ & $\begin{array}{c}12.0 \\
9\end{array}$ & $\begin{array}{c}10.6 \\
37\end{array}$ & $\begin{array}{c}11.5 \\
15\end{array}$ & $\begin{array}{c}10.8 \\
17\end{array}$ & $\begin{array}{l}110 \\
128\end{array}$ & $\begin{array}{c}10.3 \\
23\end{array}$ & $\begin{array}{c}11.0 \\
73\end{array}$ & $\begin{array}{c}10.7 \\
77\end{array}$ & $\begin{array}{c}10.4 \\
48\end{array}$ \\
\hline $\mathrm{Al}$ & 4.95 & $\begin{array}{c}4.73 \\
4\end{array}$ & $\begin{array}{c}4.82 \\
5\end{array}$ & $\begin{array}{c}4.19 \\
4\end{array}$ & $\begin{array}{c}4.49 \\
4\end{array}$ & $\begin{array}{c}4.04 \\
6\end{array}$ & $\begin{array}{c}4.66 \\
3\end{array}$ & $\begin{array}{c}4.53 \\
3\end{array}$ & 4.86 & $\begin{array}{c}4.54 \\
9\end{array}$ & $\begin{array}{c}4.50 \\
4\end{array}$ & $\begin{array}{c}4.84 \\
3\end{array}$ \\
\hline $\mathrm{Fe}_{3}$ & $\begin{array}{c}0.05 \\
8\end{array}$ & $\begin{array}{c}0.05 \\
9\end{array}$ & $\begin{array}{c}0.05 \\
7\end{array}$ & $\begin{array}{c}0.03 \\
6\end{array}$ & $\begin{array}{c}0.05 \\
5\end{array}$ & $\begin{array}{c}0.01 \\
8\end{array}$ & $\begin{array}{c}0.03 \\
1\end{array}$ & 0.03 & $\begin{array}{c}0.06 \\
1\end{array}$ & $\begin{array}{c}0.03 \\
4\end{array}$ & $\begin{array}{c}0.02 \\
3\end{array}$ & $\begin{array}{c}0.04 \\
3\end{array}$ \\
\hline $\mathrm{Ti}$ & 0 & 0 & 0 & 0 & 0 & 0 & 0 & 0 & 0 & 0 & 0 & 0 \\
\hline $\mathrm{Na}$ & $\begin{array}{c}1.50 \\
9\end{array}$ & 0 & $\begin{array}{c}2.59 \\
5\end{array}$ & $\begin{array}{c}1.00 \\
8\end{array}$ & $\begin{array}{c}3.05 \\
7\end{array}$ & $\begin{array}{c}2.10 \\
2\end{array}$ & $\begin{array}{c}2.20 \\
5\end{array}$ & 1.88 & $\begin{array}{c}2.04 \\
6\end{array}$ & $\begin{array}{c}1.71 \\
7\end{array}$ & $\begin{array}{c}2.42 \\
7\end{array}$ & $\begin{array}{c}2.83 \\
9\end{array}$ \\
\hline $\mathrm{Ca}$ & $\begin{array}{c}2.51 \\
6\end{array}$ & $\begin{array}{c}2.69 \\
3\end{array}$ & $\begin{array}{c}2.28 \\
2\end{array}$ & $\begin{array}{c}0.89 \\
7\end{array}$ & $\begin{array}{c}2.32 \\
1\end{array}$ & $\begin{array}{c}1.77 \\
6\end{array}$ & $\begin{array}{c}2.17 \\
7\end{array}$ & $\begin{array}{c}1.92 \\
2\end{array}$ & $\begin{array}{c}2.93 \\
4\end{array}$ & $\begin{array}{c}2.08 \\
9\end{array}$ & 2.36 & $\begin{array}{c}2.28 \\
3\end{array}$ \\
\hline $\mathrm{K}$ & $\begin{array}{c}0.10 \\
8\end{array}$ & $\begin{array}{c}0.20 \\
4\end{array}$ & $\begin{array}{c}0.15 \\
5\end{array}$ & $\begin{array}{c}0.13 \\
4\end{array}$ & $\begin{array}{c}0.09 \\
1\end{array}$ & $\begin{array}{c}0.08 \\
3\end{array}$ & $\begin{array}{c}0.07 \\
6\end{array}$ & $\begin{array}{c}0.06 \\
4\end{array}$ & $\begin{array}{c}0.04 \\
7\end{array}$ & $\begin{array}{c}0.05 \\
2\end{array}$ & $\begin{array}{c}0.14 \\
8\end{array}$ & 0.13 \\
\hline $\mathrm{Al} \%$ & 36.5 & 0 & 51.6 & 49.4 & 55.9 & 53.1 & 49.5 & 48.6 & 40.7 & 44.5 & 49.2 & 54.1 \\
\hline $\begin{array}{c}\mathrm{An} \\
\%\end{array}$ & 60.9 & 93 & 45.3 & 44 & 42.4 & 44.8 & 48.8 & 49.7 & 58.4 & 54.1 & 47.8 & 43.5 \\
\hline Or\% & 2.6 & 7 & 3.1 & 6.6 & 1.7 & 2.1 & 1.7 & 1.7 & 0.9 & 1.3 & 3 & 2.5 \\
\hline
\end{tabular}

Table 3 (cont.) 


\begin{tabular}{|c|c|c|c|c|c|c|c|c|c|c|c|c|}
\hline Sample & $\begin{array}{c}\mathrm{A} 154- \\
5\end{array}$ & $\begin{array}{c}\mathrm{A} 154- \\
6\end{array}$ & $\begin{array}{c}\mathrm{A} 154- \\
7\end{array}$ & $\begin{array}{c}\mathrm{A} 178- \\
1\end{array}$ & $\begin{array}{c}\mathrm{A} 178- \\
\mathrm{A} 178-\end{array}$ & 5 & $\mathrm{~A} 178-$ & $\mathrm{A} 178-$ & $\mathrm{A} 234-$ & $\mathrm{A} 234-$ & $\mathrm{A} 239-$ & $\mathrm{A} 239-$ \\
2 & & 2 & 5 & 1 & 2 \\
\hline $\mathrm{SiO}_{2}$ & 58.42 & 60.84 & 59.09 & 44.94 & 45.29 & 44.67 & 45.03 & 45.82 & 58.57 & 62.55 & 48.08 & 49 \\
\hline $\mathrm{Al}_{2} \mathrm{O}_{3}$ & 19.36 & 22.42 & 20.31 & 28.34 & 27.94 & 27.19 & 26.65 & 27.84 & 21.73 & 20.91 & 27.51 & 26.37 \\
\hline $\mathrm{Fe}_{2} \mathrm{O}_{3}$ & 0.28 & 0.34 & 0.29 & 0.6 & 0.63 & 0.56 & 0.62 & 0.62 & 0.34 & 0.5 & 0.48 & 0.52 \\
\hline $\mathrm{TiO}_{2}$ & - & - & - & - & - & - & - & - & - & - & - & - \\
\hline $\mathrm{Na}_{2} \mathrm{O}$ & 7.06 & 2.71 & 10.24 & 1.41 & - & 2.06 & 3.42 & 1.31 & 7.88 & 5.06 & - & - \\
\hline $\mathrm{CaO}$ & 11.45 & 13.09 & 9.53 & 24.66 & 26.05 & 25.46 & 23.99 & 24.16 & 11.02 & 8.81 & 23.62 & 23.89 \\
\hline $\mathrm{K}_{2} \mathrm{O}$ & 0.47 & 0.55 & 0.51 & - & - & - & 0.15 & 0.16 & 0.33 & 1.74 & 0.15 & 0.14 \\
\hline $\mathrm{MnO}$ & - & - & - & - & - & - & - & - & - & - & 0.06 & 0.01 \\
\hline $\mathrm{Total}$ & 97.1 & 99.95 & 99.97 & 99.95 & 99.91 & 99.94 & 99.86 & 99.91 & 99.87 & 99.57 & 99.84 & 99.92 \\
\hline $\mathrm{Si}$ & 10.945 & 10.894 & 10.81 & 8.537 & 8.596 & 8.543 & 8.629 & 8.685 & 11.158 & 11.239 & 9.008 & 9.175 \\
\hline $\mathrm{Al}$ & 4.272 & 4.728 & 4.378 & 6.34 & 6.245 & 6.124 & 6.014 & 6.214 & 4.875 & 4.425 & 6.07 & 5.815 \\
\hline $\mathrm{Fe}$ & 0.039 & 0.046 & 0.04 & 0.086 & 0.09 & 0.081 & 0.089 & 0.088 & 0.049 & 0.068 & 0.068 & 0.073 \\
\hline $\mathrm{Ti}$ & 0 & 0 & 0 & 0 & 0 & 0 & 0 & 0 & 0 & 0 & 0 & 0 \\
\hline $\mathrm{Na}$ & 2.565 & 0.941 & 3.634 & 0.519 & 0 & 0.764 & 1.271 & 0.481 & 0 & 1.763 & 0 & 0 \\
\hline $\mathrm{Ca}$ & 2.298 & 2.511 & 1.869 & 5.019 & 5.297 & 5.217 & 4.925 & 4.906 & 2.249 & 1.696 & 4.741 & 4.793 \\
\hline
\end{tabular}




\begin{tabular}{|c|c|c|c|c|c|c|c|c|c|c|c|c|}
\hline $\mathrm{K}$ & 0.112 & 0.126 & 0.119 & 0 & 0 & 0 & 0.037 & 0.039 & 0.08 & 0.399 & 0.036 & 0.033 \\
\hline $\mathrm{Al} \%$ & 51.6 & 26.3 & 64.6 & 9.4 & 0 & 12.8 & 20.4 & 8.9 & 0 & 45.7 & 0 & 0 \\
\hline $\mathrm{An} \%$ & 46.2 & 70.2 & 33.2 & 90.6 & 100 & 87.2 & 79 & 90.4 & 96.6 & 44 & 99.2 & 99.3 \\
\hline $\mathrm{Or} \%$ & 2.3 & 3.5 & 2.1 & 0 & 0 & 0 & 0.6 & 0.7 & 3.4 & 10.3 & 0.8 & 0.7 \\
\hline
\end{tabular}

Table 3 (cont.)

\begin{tabular}{|c|c|c|c|c|c|c|c|c|c|c|c|c|}
\hline Sample & $\begin{array}{c}\mathrm{A} 239- \\
3\end{array}$ & $\begin{array}{c}\mathrm{A} 239- \\
4\end{array}$ & $\begin{array}{c}\mathrm{A} 239- \\
9\end{array}$ & $\begin{array}{c}\mathrm{A} 240- \\
6\end{array}$ & $\begin{array}{c}\mathrm{A} 240- \\
8\end{array}$ & $\begin{array}{c}\mathrm{A} 240- \\
\mathrm{A} 247-\end{array}$ & $\begin{array}{c}\mathrm{A} 247- \\
6\end{array}$ & $\begin{array}{c}\mathrm{A} 250- \\
1\end{array}$ & $\begin{array}{c}\mathrm{A} 250- \\
4\end{array}$ & $\begin{array}{c}\mathrm{A} 250- \\
5\end{array}$ & $\begin{array}{c}\mathrm{A} 250- \\
8\end{array}$ \\
\hline $\mathrm{SiO}_{2}$ & 49.95 & 47.56 & 57.6 & 48.41 & 48.65 & 50.71 & 57.48 & 63.44 & 60.59 & 50.15 & 48.83 & 58.14 \\
\hline $\mathrm{Al}_{2} \mathrm{O}_{3}$ & 26.5 & 27.28 & 22.5 & $27 / 81$ & 26.25 & 26.29 & 22.53 & 21.97 & 20.04 & 26.85 & 26.43 & 23.68 \\
\hline $\mathrm{Fe}_{2} \mathrm{O}_{3}$ & 0.74 & 0.64 & 0.57 & 0.71 & 0.51 & 0.76 & 0.34 & 0.44 & 0.39 & 0.62 & 0.47 & 0.41 \\
\hline $\mathrm{TiO}_{2}$ & 0.45 & 0.01 & 0.09 & - & - & - & 0.11 & 0.14 & - & - & - & 0.16 \\
\hline $\mathrm{Na}_{2} \mathrm{O}$ & - & - & 1.03 & - & - & - & 6.44 & - & 5.91 & - & - & - \\
\hline $\mathrm{CaO}$ & 22.17 & 24.34 & 17.77 & 22.74 & 24.2 & 21.86 & 12.26 & 13.6 & 12.29 & 22.31 & 24.18 & 16.84 \\
\hline $\mathrm{K} 2 \mathrm{O}$ & 0.13 & 0.1 & 0.38 & 0.16 & 0.17 & 0.18 & 0.41 & 0.35 & 0.7 & - & 0.09 & 0.42 \\
\hline $\mathrm{MnO}$ & - & - & - & 0.08 & - & - & - & - & - & - & - & - \\
\hline $\mathrm{Total}$ & 99.94 & 99.93 & 99.94 & 99.91 & 99.78 & 99.80 & 99.57 & 99.94 & 99.92 & 99.93 & 100 & 99.65 \\
\hline $\mathrm{Si}$ & 9.287 & 8.94 & 10.53 & 9.042 & 9.14 & 9.418 & 10.511 & 11.202 & 10.991 & 9.31 & 9.144 & 10.519 \\
\hline $\mathrm{Al}$ & 5.803 & 6.039 & 4.844 & 6.117 & 5.808 & 5.75 & 4.852 & 4.568 & 4.281 & 5.87 & 5.828 & 5.046 \\
\hline
\end{tabular}




\begin{tabular}{|c|c|c|c|c|c|c|c|c|c|c|c|c|}
\hline $\mathrm{Fe}_{3}$ & 0.103 & 0.09 & 0.078 & 0.1 & 0.072 & 0.106 & 0.047 & 0.058 & 0.053 & 0.087 & 0.066 & 0.019 \\
\hline $\mathrm{Ti}$ & 0.063 & 0.001 & 0.012 & 0 & 0 & 0 & 0.015 & 0.019 & 0 & 0 & 0 & 0.022 \\
\hline $\mathrm{Na}$ & 0 & 0 & 0 & 0 & 0 & 0 & 2.284 & 0 & 2.079 & 0 & 0 & 0 \\
\hline $\mathrm{Ca}$ & 4.416 & 4.902 & 3.481 & 4.551 & 4.871 & 4.35 & 2.402 & 2.573 & 2.389 & 4.437 & 4.851 & 3.265 \\
\hline $\mathrm{K}$ & 0.031 & 0.024 & 0.089 & 0.038 & 0.041 & 0.043 & 0.096 & 0.079 & 0.162 & 0 & 0.022 & 0.097 \\
\hline $\mathrm{Al} \%$ & 0 & 0 & 0 & 0 & 0 & 0 & 47.8 & 0 & 44.9 & 0 & 0 & 0 \\
\hline $\mathrm{An} \%$ & 99.3 & 99.5 & 97.5 & 99.2 & 99.2 & 99 & 50.2 & 97 & 51.6 & 100 & 99.5 & 97.1 \\
\hline $\mathrm{Or} \%$ & 0.7 & 0.5 & 2.5 & 0.8 & 0.8 & 1 & 2 & 3 & 3.5 & 0 & 0.5 & 2.9 \\
\hline
\end{tabular}

Table 4. Microprobe analysis results for the amphibole from the rocks in the study region.

\begin{tabular}{|c|c|c|c|c|c|c|c|c|c|c|c|c|c|c|c|c|}
\hline $\begin{array}{c}\text { Sampl } \\
\mathrm{e}\end{array}$ & A6-1 & A6-4 & $\begin{array}{c}\text { A23- } \\
5\end{array}$ & $\begin{array}{c}\text { A23- } \\
6\end{array}$ & $\begin{array}{c}\text { A134 } \\
-1\end{array}$ & $\begin{array}{c}\text { A154 } \\
-2\end{array}$ & $\begin{array}{c}\text { A154 } \\
-3\end{array}$ & $\begin{array}{c}\text { A178 } \\
-3\end{array}$ & $\begin{array}{c}\text { A178 } \\
-4\end{array}$ & $\begin{array}{c}\mathrm{A} 234 \\
-1\end{array}$ & $\begin{array}{c}\mathrm{A} 234 \\
-3\end{array}$ & $\begin{array}{c}\text { A234 } \\
-4\end{array}$ & $\begin{array}{c}\text { A239 } \\
-6\end{array}$ & $\begin{array}{c}\text { A239 } \\
-7\end{array}$ & $\begin{array}{c}\mathrm{A} 250 \\
-2\end{array}$ & $\begin{array}{c}\text { A250 } \\
-6\end{array}$ \\
\hline $\mathrm{SiO}_{2}$ & 43.61 & 45.72 & 43.25 & 42.74 & 38.43 & 47.07 & 44.7 & 43.26 & 45.14 & 34.08 & 42.32 & 40.37 & 38.79 & 37.06 & 35.76 & 37.23 \\
\hline $\mathrm{TiO}_{2}$ & 1.74 & 0.93 & 1.53 & 2.02 & 2.56 & 1.65 & 1.77 & 2.43 & 1.38 & 6.24 & 2.41 & 4.15 & 6.35 & 6.8 & 6.67 & 6 \\
\hline $\mathrm{Al}_{2} \mathrm{O}_{3}$ & 4.66 & 2.86 & 3.54 & 5.07 & 5.32 & 6.25 & 3.96 & 2.39 & 2.04 & 11.64 & 6.77 & 8.71 & 8.09 & 7.86 & 6.88 & 7.48 \\
\hline $\mathrm{FeO}$ & 21.65 & 21.68 & 23.45 & 24.12 & 33.72 & 18.29 & 20.77 & 12.44 & 10.67 & 28.3 & 21.64 & 18.95 & 20.95 & 24.29 & 25.86 & 23.77 \\
\hline $\mathrm{Cr}_{2} \mathrm{O}_{3}$ & 0.05 & 0.08 & 0.07 & - & - & - & - & - & - & 0.14 & 0.06 & 0.08 & - & - & - & - \\
\hline $\mathrm{MnO}$ & 0.78 & 0.94 & 0.78 & 0.88 & 1.11 & 0.44 & 0.47 & 0.27 & 0.27 & 0.43 & 0.49 & 0.37 & 0.34 & 0.4 & 0.4 & 0.49 \\
\hline
\end{tabular}




\begin{tabular}{|c|c|c|c|c|c|c|c|c|c|c|c|c|c|c|c|c|}
\hline $\mathrm{MgO}$ & 11.65 & 12.28 & 10.82 & 10.29 & 4.8 & 9 & 12.04 & 10.23 & 11.74 & 10.93 & 10.34 & 10.78 & 10.1 & 8.74 & 8.81 & 9.39 \\
\hline $\mathrm{CaO}$ & 14.1 & 14.87 & 15.96 & 13.62 & 12.15 & 14.35 & 15.44 & 28.7 & 28.54 & 7.75 & 14.88 & 15.52 & 14.24 & 13.59 & 14.36 & 14.15 \\
\hline $\mathrm{Na}_{2}$ & - & - & - & - & - & - & - & - & - & - & - & - & . & - & - & - \\
\hline $\mathrm{K}_{2} \mathrm{O}$ & 1.67 & 0.48 & 0.53 & 1.18 & 1.08 & 2.85 & 0.61 & - & - & 0.35 & 0.78 & 0.96 & 0.91 & 0.88 & 1.11 & 1.17 \\
\hline Total & 99.91 & 99.84 & 99.93 & 99.92 & 99.17 & 99.90 & 99.76 & 99.72 & 99.79 & 99.86 & 99.69 & 99.89 & 99.77 & 99.62 & 99.85 & 99.68 \\
\hline $\mathrm{Si}$ & 6.49 & 6.741 & 6.482 & 6.383 & 6.017 & 7.135 & 6.619 & 6.915 & 7.099 & 4.909 & 6.307 & 5.999 & 5.808 & 5.619 & 5.467 & 5.644 \\
\hline $\mathrm{Ti}$ & 0 & 0 & 0.088 & 0 & 0 & 0 & 0.1 & 0 & 0 & 0 & 0 & 0.236 & 0.361 & 0 & 0.388 & 0 \\
\hline $\mathrm{Al}$ & 0.817 & 0.497 & 0.625 & 0.892 & 0.981 & 0.865 & 0.691 & 0.391 & 0.27 & 1.975 & 1.188 & 1.524 & 1.426 & 1.403 & 1.239 & 1.335 \\
\hline $\mathrm{Fe}$ & 1.473 & 1.336 & 1.584 & 1.543 & 2.29 & 2.319 & 1.498 & 0.916 & 0.771 & 0.199 & 1.602 & 1.508 & 1.576 & 1.666 & 1.611 & 1.556 \\
\hline $\mathrm{Cr}$ & 0.006 & 0.009 & 0.008 & 0 & 0 & 0 & 0 & 0 & 0 & 0.016 & 0.007 & 0.009 & 0 & 0 & 0 & 0 \\
\hline $\mathrm{Mn}$ & 0.098 & 0.117 & 0.049 & 0.111 & 0.147 & 0.056 & 0.049 & 0.037 & 0.036 & 0.025 & 0.062 & 0.047 & 0.043 & 0.051 & 0.052 & 0.063 \\
\hline $\mathrm{Mg}$ & 2.585 & 2.699 & 2.418 & 2.291 & 1.12 & 2.034 & 2.658 & 2.438 & 2.753 & 2.347 & 2.297 & 2.388 & 2.254 & 1.975 & 2.008 & 2.122 \\
\hline $\mathrm{Ca}$ & 0.114 & 0.16 & 0.256 & 0.083 & 0.018 & 0.152 & 0.205 & 1.206 & 1.167 & 0 & 0.172 & 0.32 & 0.441 & 0.096 & 0.514 & 0.137 \\
\hline $\mathrm{Na}$ & 0 & 0 & 0 & 0 & 0 & 0 & 0 & 0 & 0 & 0 & 0 & 0 & 0 & 0 & 0 & 0 \\
\hline $\mathrm{K}$ & 0.317 & 0.09 & 0.101 & 0.225 & 0.216 & 0.551 & 0.115 & 0 & 0 & 0.064 & 0.148 & 0.182 & 0.174 & 0.17 & 0.216 & 0.226 \\
\hline $\begin{array}{c}\text { Sum- } \\
\text { cat }\end{array}$ & $\begin{array}{c}15.45 \\
1\end{array}$ & $\begin{array}{c}15.27 \\
9\end{array}$ & $\begin{array}{c}15.40 \\
9\end{array}$ & $\begin{array}{c}15.32 \\
2\end{array}$ & $\begin{array}{c}15.23 \\
6\end{array}$ & 15.73 & 15.36 & $\begin{array}{c}16.70 \\
9\end{array}$ & $\begin{array}{c}16.64 \\
2\end{array}$ & $\begin{array}{c}14.64 \\
5\end{array}$ & $\begin{array}{c}15.35 \\
2\end{array}$ & $\begin{array}{c}15.43 \\
8\end{array}$ & $\begin{array}{c}15.32 \\
8\end{array}$ & $\begin{array}{c}15.28 \\
2\end{array}$ & $\begin{array}{c}15.40 \\
7\end{array}$ & $\begin{array}{c}15.38 \\
8\end{array}$ \\
\hline
\end{tabular}




$$
\text { V. } 8 \text { - No } 03 \text { - Ano } 2019
$$

ISSN | 2179-7137 | http://periodicos.ufpb.br/ojs2/index.php/ged/index

Table 5. Microprobe analysis results for the pyroxene from the rocks in the study region.

\begin{tabular}{|c|c|c|c|c|c|c|c|c|c|}
\hline Sample & $\mathrm{A} 239-5$ & $\mathrm{~A} 239-8$ & $\mathrm{~A} 240-3$ & $\mathrm{~A} 240-4$ & $\mathrm{~A} 240-7$ & $\mathrm{~A} 247-1$ & $\mathrm{~A} 247-2$ & $\mathrm{~A} 250-3$ & $\mathrm{~A} 250-7$ \\
\hline $\mathrm{SiO}_{2}$ & 44.86 & 46.06 & 44.42 & 43.39 & 43.01 & 44.43 & 44.66 & 42.47 & 43.83 \\
\hline $\mathrm{Al}_{2} \mathrm{O}_{3}$ & 1.38 & 1.4 & 1.27 & 2.23 & 1.92 & 1.17 & 1.47 & 2.1 & 1.5 \\
\hline $\mathrm{Fe}_{2} \mathrm{O}_{3}$ & 13.41 & 13.24 & 16.81 & 14.07 & 14.4 & 16.16 & 17.24 & 18.41 & 16.55 \\
\hline $\mathrm{TiO}_{2}$ & 1.1 & 1.01 & 0.93 & 1.73 & 1.29 & 1.14 & 1.26 & 1.55 & 1.3 \\
\hline $\mathrm{Na}_{2} \mathrm{O}$ & - & - & - & - & - & - & - & - & - \\
\hline $\mathrm{CaO}$ & 27.17 & 26.44 & 26 & 25.45 & 26.97 & 27.3 & 25.26 & 23.94 & 25.29 \\
\hline $\mathrm{K} 2 \mathrm{O}$ & - & - & - & 0.18 & - & - & - & - & 0.18 \\
\hline $\mathrm{FeO}$ & - & - & - & - & - & - & - & - & - \\
\hline $\mathrm{MnO}$ & 0.48 & 0.51 & 0.63 & 0.44 & 0.44 & 0.43 & 0.62 & 0.55 & 0.59 \\
\hline $\mathrm{MgO}$ & 11.6 & 11.18 & 9.85 & 12.26 & 11.9 & 9.26 & 9.4 & 10.7 & 10.76 \\
\hline $\mathrm{Cr} 2 \mathrm{O}_{3}$ & - & - & - & - & - & - & - & - & - \\
\hline $\mathrm{NiO}$ & - & - & - & - & - & - & - & - & - \\
\hline $\mathrm{Total}$ & 100 & 99.84 & 99.91 & 99.75 & 99.93 & 99.89 & 99.91 & 99.72 & 100 \\
\hline $\mathrm{Si}$ & 1.722 & 1.774 & 1.241 & 1.655 & 1.653 & 1.738 & 1.753 & 1.665 & 1.17 \\
\hline $\mathrm{Al}$ & 0.062 & 0.064 & 0.042 & 0.1 & 0.087 & 0.054 & 0.068 & 0.097 & 0.047 \\
\hline
\end{tabular}




$$
\text { V. } 8 \text { - No } 03 \text { - Ano } 2019
$$

ISSN | 2179-7137 | http://periodicos.ufpb.br/ojs2/index.php/ged/index

\begin{tabular}{|c|c|c|c|c|c|c|c|c|c|}
\hline $\mathrm{Fe}_{2}$ & 0.216 & 0.163 & 0.393 & 0.245 & 0.26 & 0.208 & 0.179 & 0.238 & 0.369 \\
\hline $\mathrm{Ti}$ & 0.032 & 0.03 & 0.02 & 0.05 & 0.037 & 0.034 & 0.037 & 0.046 & 0.026 \\
\hline $\mathrm{Na}$ & 0 & 0 & 0 & 0 & 0 & 0 & 0 & 0 & 0 \\
\hline $\mathrm{Ca}$ & 1.117 & 1.091 & 0.599 & 1.081 & 1.111 & 1.144 & 1.062 & 1.006 & 0.723 \\
\hline $\mathrm{K}$ & 0 & 0 & 0 & 0 & 0 & 0 & 0 & 0 & 0 \\
\hline $\mathrm{Fe}$ & 0 & 0 & 0 & 0 & 0 & 0 & 0 & 0 & 0 \\
\hline $\mathrm{Mn}$ & 0.016 & 0.017 & 0.015 & 0.014 & 0.014 & 0.014 & 0.021 & 0.018 & 0.013 \\
\hline $\mathrm{Mg}$ & 0.664 & 0.642 & 0.41 & 0.697 & 0.682 & 0.54 & 0.55 & 0.625 & 0.428 \\
\hline $\mathrm{Cr}$ & 0 & 0 & 0 & 0 & 0 & 0 & 0 & 0 & 0 \\
\hline $\mathrm{Ni}$ & 0 & 0 & 0 & 0 & 0 & 0 & 0 & 0 & 0 \\
\hline $\mathrm{Wo} \%$ & 51.169 & 51.152 & 52.586 & 49.229 & 49.964 & 52.639 & 49.602 & 45.88 & 48.245 \\
\hline $\mathrm{En} \%$ & 30.397 & 30.095 & 25.086 & 31.749 & 30.674 & 24.843 & 25.683 & 28.532 & 30.023 \\
\hline $\mathrm{Fs} \%$ & 18.435 & 18.752 & 21.687 & 19.021 & 19.362 & 22.518 & 24.715 & 25.588 & 21.217 \\
\hline
\end{tabular}

Table 6. Microprobe analysis results for the olivine from the rocks in the study region.

\begin{tabular}{|c|c|c|c|c|c|c|c|}
\hline Semple & $\mathrm{A} 239-10$ & $\mathrm{~A} 239-11$ & $\mathrm{~A} 240-1$ & $\mathrm{~A} 240-2$ & $\mathrm{~A} 240-5$ & $\mathrm{~A} 247-3$ & A247-4 \\
\hline $\mathrm{SiO}_{2}$ & 26.84 & 26.33 & 28.36 & 30.21 & 30.32 & 32.21 & 27.56 \\
\hline $\mathrm{TiO}_{2}$ & - & - & - & - & - & - & - \\
\hline
\end{tabular}




\begin{tabular}{|c|c|c|c|c|c|c|c|}
\hline $\mathrm{Al}_{2} \mathrm{O}_{3}$ & - & - & - & - & - & - & - \\
\hline $\mathrm{FeO}$ & 50.78 & 53.63 & 43.98 & 41.38 & 41.66 & 40.2 & 55.75 \\
\hline $\mathrm{MnO}$ & 1.06 & 1.14 & 0.71 & 0.77 & 0.75 & 0.77 & 1.13 \\
\hline $\mathrm{MgO}$ & 21.15 & 18.76 & 26.77 & 27.64 & 27.16 & 26.63 & 15.36 \\
\hline $\mathrm{CaO}$ & 0.06 & 0.07 & 0.06 & - & 0.04 & 0.09 & 0.09 \\
\hline $\mathrm{Na}_{2} \mathrm{O}$ & - & - & - & - & - & - & - \\
\hline $\mathrm{K}_{2} \mathrm{O}$ & - & - & - & - & - & - & - \\
\hline $\mathrm{NiO}$ & - & - & - & - & - & - & - \\
\hline Total & 99.89 & 99.93 & 99.88 & 100 & 99.93 & 99.901 & 99.89 \\
\hline $\mathrm{Si}$ & 0.835 & 0.832 & 0.846 & 0.883 & 0.888 & 1.252 & 1.18 \\
\hline $\mathrm{Ti}$ & 0 & 0 & 0 & 0 & 0 & 0 & 0 \\
\hline $\mathrm{Al}$ & 0 & 0 & 0 & 0 & 0 & 0 & 0 \\
\hline $\mathrm{Fe}_{2}$ & 1.321 & 1.418 & 1.097 & 1.011 & 1.02 & 0.748 & 0.82 \\
\hline $\mathrm{Mn}$ & 0.028 & 0.031 & 0.018 & 0.019 & 0.019 & 0.025 & 0.041 \\
\hline $\mathrm{Mg}$ & 0.98 & 0.884 & 1.191 & 1.204 & 1.185 & 0.573 & 0.026 \\
\hline $\mathrm{Ca}$ & 0.002 & 0.002 & 0.002 & 0 & 0.001 & 0.004 & 0.004 \\
\hline $\mathrm{Na}$ & 0 & 0 & 0 & 0 & 0 & 0 & 0 \\
\hline
\end{tabular}




\begin{tabular}{|c|c|c|c|c|c|c|c|}
\hline $\mathrm{K}$ & 0 & 0 & 0 & 0 & 0 & 0 & 0 \\
\hline $\mathrm{Ni}$ & 0 & 0 & 0 & 0 & 0 & 0 & 0 \\
\hline Cations & 3.166 & 3.167 & 3.154 & 3.117 & 3.113 & 3.116 & 3.123 \\
\hline $\mathrm{Fe}-\mathrm{FeMg}$ & 0.57 & 0.62 & 0.48 & 0.46 & 0.46 & 0.49 & 0.46 \\
\hline $\mathrm{Mg}-\mathrm{FeMg}$ & 0.43 & 0.38 & 0.52 & 0.54 & 0.54 & 0.51 & 0.54 \\
\hline
\end{tabular}




\section{References}

Agemar, T., Wörner, G., \& Heumann, A. (1999). Stable isotopes and amphibole chemistry on hydrothermally altered granitoids in the North Chilean Precordillera: a limited role for meteoric water?. Contributions to Mineralogy and Petrology, 136(4), 331-344.

Aghanabati, A. (2004). Geology of Iran. Geological survey of Iran, Tehran (in Persian).

Alavi, M. (1994). Tectonics of the Zagros orogenic belt of Iran: new data and

interpretations. Tectonophysics, 229(34), 211-238.

Alavi, M. (2004). Regional stratigraphy of the Zagros fold-thrust belt of Iran and its proforeland evolution. American journal of science, 304(1), 1-20.

Berberian, M., \& King, G. C. P. (1981). Towards a paleogeography and tectonic evolution of Iran. Canadian journal of earth sciences, 18(2), 210-265.

Boynton, W. V. (1984). Cosmochemistry of the rare earth elements: meteorite studies. In Developments in geochemistry (Vol. 2, pp. 63-114). Elsevier.
Chappell, B. W., \& White, A. J. (2001). Two contrasting granite types: 25 years later. Australian journal of earth sciences, 48(4), 489-499.

Chivas, A. R. (1982). Geochemical evidence for magmatic fluids in porphyry copper mineralization. Contributions to Mineralogy and Petrology, 78(4), 389403.

Cox, K. G. BELL, JD: PANKHURST, RJ (1979) The interpretation of igneous rocks. William Clowes, London, Britain. Deer, W., Howie, R., \& Zussman, J. (1992). An introduction to the rockforming minerals: Essex. England: Longman Scientific and Technology. Ghalamghash, J., Nédélec, A., Bellon, H., Abedini, M. V., \& Bouchez, J. L. (2009). The Urumieh plutonic complex (NW Iran): A record of the geodynamic evolution of the Sanandaj-Sirjan zone during Cretaceous times-Part I: Petrogenesis and K/Ar dating. Journal of Asian Earth Sciences, 35(5), 401-415.

Ghasemi, A., \& Talbot, C. J. (2006). A new tectonic scenario for the SanandajSirjan Zone (Iran). Journal of Asian Earth Sciences, 26(6), 683-693.

Frost, B. R., Barnes, C. G., Collins, W. J., Arculus, R. J., Ellis, D. J., \& Frost, C. D. (2001). A geochemical classification 
for granitic rocks. Journal of petrology, 42(11), 2033-2048.

Hassanzadeh, J., Stockli, D. F., Horton, B. K., Axen, G. J., Stockli, L. D., Grove, M., .. \& Walker, J. D. (2008). U-Pb zircon geochronology of late Neoproterozoic-Early Cambrian granitoids in Iran: Implications for paleogeography, magmatism, and exhumation history of Iranian basement. Tectonophysics, 451(1-4), $71-96$.

Hawthorne, F. C. (1983). The crystal chemistry of the amphiboles; Appendices. The Canadian

Mineralogist, 21(2), 353-480.

Khalaji, A. A., Esmaeily, D., Valizadeh, M. V., \& Rahimpour-Bonab, H. (2007). Petrology and geochemistry of the granitoid complex of Boroujerd, Sanandaj-Sirjan Zone, Western Iran. Journal of Asian Earth Sciences, 29(5-6), 859-877.

Khodami, M., Kamali Shervedani, A (2018) Mineralogical and geochemical characteristics of the Chah-Shur clay deposit, Southeast of Isfahan, Iran, Iranian Journal of Earth Sciences, 10(2): $135-141$.

Leake, B. E., Woolley, A. R., Arps, C.

E., Birch, W. D., Gilbert, M. C., Grice, J. D., $\quad \ldots \quad \& \quad$ Linthout, K. (1997).
Nomenclature of Amphiboles: Report of the Subcommitte on Amphiboles of the International Mineralogical Association Commision on New Minerals and Mineral Names (3). Canadian Mineralogist, 35(1), 219-248.

Maniar, P. D., \& Piccoli, P. M. (1989). Tectonic discrimination of granitoids. Geological society of America bulletin, 101(5), 635-643.

Mazhari, S. A., Bea, F., Amini, S., Ghalamghash, J., Molina, J. F., Montero, P., .. \& Williams, I. S. (2009). The Eocene bimodal Piranshahr massif of the Sanandaj-Sirjan Zone, NW Iran: a marker of the end of the collision in the Zagros orogen. Journal of the Geological Society, 166(1), 53-69.

Mobashergarmi, M., Zaraisahamia, R., Aghazadeh, M., Ahmadikhalaji, A., Ahmadzadeh, GH (2018) Mineral chemistry and thermobarometry of Eocene alkaline volcanic rocks in SW Germi, NW Iran, Iranian Journal of Earth Sciences 10 (1) 39-51.

Mohajjel, M., \& Fergusson, C. L. (2000). Dextral transpression in Late Cretaceous continental collision, Sanandaj-Sirjan zone, western Iran. Journal of structural geology, 22(8), 1125-1139.

Mohajjel, M., Fergusson, C. L., \& Sahandi, M. R. (2003). Cretaceous- 
Tertiary convergence and continental collision, Sanandaj-Sirjan zone, western Iran. Journal of Asian Earth Sciences, 21(4), 397-412.

Morimoto, N. (1988). Nomenclature of pyroxenes. Mineralogy

and

Petrology, 39(1), 55-76.

Novruzov, N., Valiyev, A., Bayramov, A

., Mammadov, S., Ibrahimov, J.,

Ebdulrehimli, A (2019) Mineral composition and paragenesis of altered and mineralized zones in the Gadir low sulfidation epithermal deposit (Lesser Caucasus, Azerbaijan), Iranian Journal of Earth Sciences, 11(1): 14-29.

Numan, N. M. (2000). Discussion on

"Dextral transpression in Late Cretaceous continental collision, Sanandaj-Sirjan Zone, western Iran" [Journal of Structural Geology, 22 8) 2000) 1125ą1139]. Journal of Structural Geology, 22(8), 1125ą1139.

Omrani, J., Agard, P., Whitechurch, H., Benoit, M., Prouteau, G., \& Jolivet, L. (2008). Arc-magmatism and subduction history beneath the Zagros Mountains, Iran: a new report of adakites and geodynamic

consequences. Lithos, 106(3-4), $\quad 380-$ 398.

Peccerillo, A., \& Taylor, S. R. (1976). Geochemistry of Eocene calc-alkaline
557

volcanic rocks from the Kastamonu area, northern Turkey. Contributions to mineralogy and petrology, 58(1), 63-81. Rollinson, H. R. (1993). Using geochemical data: evaluation. Presentation, interpretation. Singapore. Ongman.

Şengör, A. M. C. (1990). A new model for the late Palaeozoic-Mesozoic tectonic evolution of Iran and implications for Oman. Geological Society, London, Special Publications, 49(1), 797-831.

Sepahi, A. A. (1999). Petrology of the Alvand plutonic complex with special reference on granitoids. PhD, TarbiatMoallem University, Tehran, Iran (in Persian).

Shahabpour, J. (2007). Island-arc affinity of the Central Iranian volcanic belt. Journal of Asian Earth Sciences, 30(5-6), 652-665.

Shand, S. J. (1943). Eruptive rocks: their genesis, composition, and classification, with a chapter on meteorites. J. Wiley \& sons, Incorporated.

Sheikholeslami, M. R., Pique, A., Mobayen, P., Sabzehei, M., Bellon, H., \& Emami, M. H. (2008). Tectonometamorphic evolution of the Neyriz metamorphic complex, Quri-kor-e-sefid area (Sanandaj-Sirjan Zone, SW 


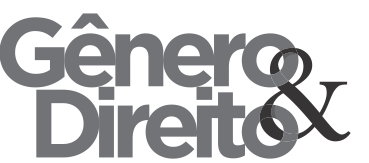

Periódico do Núcleo de Estudos e Pesquisas sobre Gênero e Direito

Centro de Ciências Jurídicas - Universidade Federal da Paraíba

V. 8 - $\mathrm{N}^{\circ} 03$ - Ano 2019

ISSN | 2179-7137 | http://periodicos.ufpb.br/ojs2/index.php/ged/index

Iran). Journal of Asian Earth

Sciences, 31(4-6), 504-521.

Stocklin, J. (1968). Structural history and tectonics of Iran: a review. AAPG bulletin, 52(7), 1229-1258.

Wood, D. A., Joron, J. L., Treuil, M., Norry, M., \& Tarney, J. (1979).

Elemental and $\mathrm{Sr}$ isotope variations in basic lavas from Iceland and the surrounding ocean floor. Contributions to Mineralogy and Petrology, 70(3), 319-339.

Yazdi, A., Ashja-Ardalan, A., Emami, M.H., Dabiri, R., \& Foudazi, M. (2017).

Chemistry of Minerals and Geothermobarometry of Volcanic Rocks in the Region Located in Southeast of Bam, Kerman Province. Open Journal of Geology, 7, 1644-1653. 